\title{
A CHARACTERIZATION OF TAME CURVES IN THREE-SPACE
}

\author{
BY \\ O. G. HARROLD, JR., H. C. GRIFFITH( $(1)$, AND E. E. POSEY
}

1. Introduction. It is one of the oldest known facts about three-dimensional topology that a simple closed curve may be knotted or unknotted. Specifically, starting with a certain curve and a class of homeomorphisms of space onto itself one asks which curves are equivalent under the class of transformations to the given one. Since such a transformation preserves not only the topological properties of the curve but also those of its complementary set, the existence of knots may be established by exhibiting curves whose complements are not homeomorphic. This was first done by means of the socalled knot-group. Later, in connection with problems dealing with the extendability of homeomorphisms given on subsets of three-space, it was discovered that a knot-group may require an infinite set of generators and that even a simple arc may be knotted in this sense. These examples, originally studied by Antoine [3], Alexander [1], and later by Fox-Artin [5], emphasize the pathological difficulties which may occur.

The purpose of the present paper is to characterize by means of positional invariants those arcs and curves which are not pathological, i.e. those sets which are tame in the sense of Fox-Artin [5]. In the language of Moise [9] our purpose may be stated to distinguish the simple closed curves that determine classical knot-types from those that do not (the pathological variety).

By the use of properties $\mathcal{P}$ and 2 (defined below) we give in Theorem VI a necessary and sufficient condition that a curve be unknotted in the sense that there exists a homeomorphism on three-space carrying the curve onto a standard circle. In $\S 8$, by the use of property $\mathcal{P}$ and a local form of property 2, we give a necessary and sufficient condition that a curve be locally tamely imbedded. By a recent result proved simultaneously by Bing [4] and Moise [10], this implies that property $P$ and the local form of property 2 are necessary and sufficient that the given curve be tamely imbedded.

As a by-product of Theorems II and III there is a generalization, the Concentric Toral Theorem (under the indicated hypotheses), to $p=1$ of a result concerning the nature of the region bounded in spherical 3 -space by a pair of disjoint polyhedral 2-manifolds of genus $p=0$.

The methods employed below are not novel. Free use is made of the results of Alexander, Graeub, and Moise. The concept of a semi-linear map

Presented to the Society, May 1, 1954; received by the editors March 29, 1954.

(1) This work was begun jointly during the tenure of a National Science Foundation Fellowship by Griffith. 
is basic and the fact the locally polyhedral character of a set is preserved by such a map is fundamental.

2. Notation and definitions. Euclidean $n$-space will be denoted by $R^{n}$. If $A$ and $B$ are any two point sets, then $A \cup B$ and $A \cap B$ will have their usual set-theoretical meanings and $A \backslash B$. will denote the set of points in $A$ but not in $B$. The null set is denoted by $\square$. The set of points whose distance from some point of $A$ is less than $\epsilon$ is denoted by $S(A, \epsilon)$. The closure of the set $A$ is denoted by $\mathrm{Cl} A$. If the set $B$ is a polyhedron, then it may also be looked upon as an integral chain so that the chain $\partial B$ and the corresponding point set $|\partial B|$ are well defined. It will be convenient, when $A$ is the image of a polyhedron $B$ under a homeomorphism $h$, to let $\partial A$ denote the point set $h(|\partial B|)$.

Following Harrold-Moise [7], the set $X \subset R^{3}$ is called locally polyhedral at $p \in R^{3}$ provided there is some closed neighborhood $N$ of $p$ in $R^{3}$ whose intersection with $X$ is null or a finite polyhedron. The set $X$ is called locally polyhedral modulo $Y(\bmod Y)$ if it is locally polyhedral at each point of $R^{3} \backslash Y$. A homeomorphism $h$ of $R^{3}$ onto $R^{3}$ is called semi-linear if and only if there are subdivisions $\sigma$ and $\tau$ of $R^{3}$ into convex cells such that every simplex of $\sigma$ is mapped affinely on some simplex of $\tau$.

Definition. Let $J$ be an arc or a simple closed curve. Then $J$ is said to have property $\mathcal{P}$ provided for each $\epsilon>0$ and point $x \in J$ there is a topological 2-sphere $K \subset S(x, \epsilon)$ which is locally polyhedral at points of $K \backslash J$, contains $x$ in its interior, and meets $J$ in a set whose cardinal is the (Menger-Urysohn) order of $x$ in $J$.

Definition. An arc (simple closed curve) $J$ is said to have property 2 provided there is a topological closed 2-cell $G$ with $J C \partial G(J=\partial G)$ which is locally polyhedral modulo $J$.

Some use will be made of the following elementary portions of the theory of linking numbers as set forth in chapter 11 of the book Topologie, by Alexandroff-Hopf [2]. The linking number of two continuous cycles $z_{1}$ and $z_{2}$ is denoted by $\nu\left(z_{1}, z_{2}\right)=\nu\left(z_{2}, z_{1}\right)$. If $z_{1}$ and $z_{2}$ are disjoint simple closed curves in $R^{3}$ and $z_{1}$ is the boundary of a disk $D$ such that $D$ and $z_{2}$ are in relative general position, then $\left|\nu\left(z_{1}, z_{2}\right)\right|$ must be zero if $D \cap z_{2}=\square$ and must be 1 if $D \cap z_{2}$ is a point.

3. Preliminaries. It is known that if $J^{*}$ is an arc contained in an arc or simple closed curve $J$ which has property $\mathcal{P}$, then $J^{*}$ also has property $\mathcal{P}$, i.e., property $\mathcal{P}$ is hereditary [8]. Also, if $J$ has property $\mathcal{P}$ it also has the enclosure property, which is to say, to each $\epsilon>0$ there is a polyhedral 2sphere or torus containing $J$ in its interior and lying in $S(J, \epsilon)$, according as $J$ is an arc or a simple closed curve [8].

Examples showing that neither property $P$ nor property 2 alone is sufficient to guarantee tame imbedding are easily constructed. Example 1.4 of $[5]$ is an arc which, although it clearly has property $\mathcal{P}$, is wildly imbedded. 
Joining the end points of this arc by a segment meeting it only at the endpoints provides a wild curve with property $P$.

Example 2.1 of the same paper is a curve which bounds a 2-cell and is not tame since the fundamental group of its complement is non-abelian. It is easily seen that the 2-cell it bounds may be taken locally polyhedral at each interior point, so this is a wild curve with property 2. Example 1.1 of that paper is readily found to be a wild arc with property 2.

Let $J$ be an arc in $R^{3}$ having properties $P$ and 2 . Then there is a disk $G$ such that $\partial G=J^{*}, J^{*} \supset J$, and $G$ is locally polyhedral mod $J$. If it can be shown that $J^{*}$ has properties $P$ and 2 , then the arc problem is reduced to the simple closed curve problem. Actually, the given $J^{*}$ clearly has property 2 since $G$ exists. Further, $J^{*}$ may possibly fail to have property $\mathcal{P}$ only if the required spheres enclosing one of the end points $a$ or $b$ of $J$ fail to exist. It will be convenient to find a new simple closed curve $J_{\infty}^{*} \supset J$ such that $J_{\infty}^{*}$ has properties $P$ and 2 . The curve $J_{\infty}^{*}$ is obtained from $J^{*}$ by a sequence of modifications of $J^{*}$.

Let $\epsilon>0$ and $K(a, \epsilon)$ be a topological 2-sphere satisfying the requirements of property $\mathcal{P}$ for $J$. Then $K(a, \epsilon) \cap J$ is a single point $x_{1}$, but the set $K(a, \epsilon)$ $\cap J^{*}$ may contain more than 2 points. If so, let $\Delta$ be a sub-disk of $K(a, \epsilon)$ which contains $x_{1}$ as an interior point and meets $J^{*}$ only at $x_{1}$. Then $\partial \Delta$ links $J^{*}$ so that $G$ and $\Delta$ satisfy the hypotheses of Lemma 5.1. In the proof of Lemma 5.1 a process will be described for constructing a disk $D_{1}$ from $\Delta$ such that $\partial \Delta=\partial D_{1}, D_{1} \cap \partial G=x_{1}$, and $D_{1} \cap G$ is the union of a finite collection of arcs which are pairwise disjoint except that some pairs may have $x_{1}$ as a common end point. Let $E_{1}=\mathrm{Cl}[K(a, \epsilon) \backslash \Delta]$ and $s_{1}=\partial E_{1}=\partial \Delta$. Then if $E_{1}$ and $D_{1}$ are in relative general position, $E_{1} \cap D_{1}$ is the union of a finite collection of mutually disjoint simple closed curves $s_{1}, s_{2}, \cdots, s_{p}, s_{p+1}, \cdots, s_{n}$. The notation is chosen so that if $D_{i}\left(E_{i}\right)$ is the sub-disk of $D_{1}\left(E_{1}\right)$ bounded by $s_{i}$, then $x_{1}$ is or is not a point of $D_{i}$ according as $i \leqq p$ or not. The number of components $s_{p+1}, \cdots, s_{n}$ may be reduced by a process similar to that described in detail (for 2-spheres) in [8]. Briefly, this iterative process consists of finding an index $j, p<j \leqq n$, such that $D_{j} \cap E_{1}=s_{j}$ and replacing $E_{1}$ by the result of deforming $\left(E_{1} \backslash E_{j}\right) \cup D_{j}$ semi-linearly away from $D_{1}$ in a neighborhood of $D_{j}$. Thus eventually a disk $E_{1}^{\prime}$ is found such that $D_{1} \cap E_{1}^{\prime}$ is $s_{1} \cup \ldots \cup s_{p}$. Then if $K_{1}$ is $D_{k} \cup E_{k}$ for that index $k$ such that $D_{k} \cap E_{1}^{\prime}=s_{k}$, $K_{1}$ is a 2 -sphere which is locally polyhedral $\bmod K_{1} \cap J=x_{1}$ and lies in $S(a, \epsilon)$ if the original $K(a, \epsilon)$ was taken in $S(a, \delta)$ for $\delta$ sufficiently small. The fact that $\partial D_{1}$ (and hence $\partial D_{k}$ ) links $J^{*}$ can be used to prove $a$ is in Int $K_{1}$. Thus if $G$ can be changed so that $K_{1}$ meets $\partial G$ in exactly two points, $K_{1}$ will satisfy the requirements of Property $\mathcal{P}$ for $a$ and the given $\epsilon$.

By the construction of $D_{1}$ (see proof of Lemma 5.1), $D_{1} \cap G$ is the union of a finite collection of arcs which are mutually disjoint except that some pairs of the collection have $x_{1}$ as a common end point. Since $D_{k}$ is a sub-disk of $D_{1}$ 
containing $x_{1}$ as an interior point, the same statement may be made regarding $D_{k} \cap G$, although $D_{k} \cap G$ may contain a larger number of arcs with both end points in its boundary than $D_{1} \cap G$. If $A$ is any arc on $G \backslash J$ which has both end points on $J^{*} \backslash J$, then $G \backslash A$ is a pair of sets whose closures are a pair of subdisks $M$ and $C$ of $G$, where $\partial C \supset(J \cup A)$ and $M \subset G \backslash J$. It is evident that such an $\operatorname{arc} A$ can be chosen so that $1^{\circ} A$ meets each of the arcs of $D_{k} \cap G$ having $x_{1}$ as one end point in a single point, and $2^{\circ} M \backslash A$ contains $E_{k} \cap G$ and all arcs of $D_{k} \cap G$ with both end points in $D_{k}$.

Then $C \cap K_{1}=C \cap D_{k}$ and consists of a finite collection of $\operatorname{arcs} P_{1}, \cdots, P_{q}$, where the end points of $P_{i}$ are $x_{1}$ and $p_{i} \in \partial C$ and $P_{i} \cap P_{j}=x_{1}, i=1, \cdots, q$. Some pair $P_{i}, P_{j}$ of these and an arc $A_{i j}$ in $K_{1}$ can be chosen so that $P_{i} \cup A_{i j} \cup P_{j}$ bounds a disk $X$ on $K_{1}$ with $X \cap C=P_{i} \cup P_{j}$. If $Y$ is the sub-disk of $C \backslash J$ bounded by $P_{i} \cup P_{j}$ and a sub-arc of $J^{*} \backslash J$, then the disk $C^{\prime}$ resulting from the semi-linear deformation of $(C \backslash Y) \cup X$ away from $K_{1}$ in a neighborhood of $X$ has at least one less arc of intersection with $K_{1}$. So after a finite number of repetitions a new disk $C_{1}$ with $J \subset \partial C_{1}$ is obtained such that $C_{1} \cap K_{1}$ is a single arc and hence $\partial C_{1} \cap K_{1}$ is a pair of points. For $K_{1}$ and $J_{1}{ }^{*}=\partial C_{1}$ we have the requirements for property $P$ fulfilled relative to this $\epsilon$. It is to be noted that the disk $M$ could be taken to lie in $S\left(a, \delta_{1}\right)$ where $\delta_{1}>0$ by starting the procedure with a 2-cell $X_{\delta}$ of $G$ whose diameter is less than $\delta_{1}$ and which contains a neighborhood of $a$ in $G$.

Thus, by choosing a sequence of $\epsilon_{i} \rightarrow 0$, a sequence of corresponding 2-cells $C_{i}$ is found, and $C=\lim _{i \rightarrow \infty} C_{i}$ is a 2-cell and has a boundary curve $J_{t}^{*} \supset J$ which satisfies property 2 and fails to have property $\mathcal{P}$ only because of the nonexistence of proper spheres enclosing $b$. A similar modification is made near $b$ to get a 2-cell $G_{\infty}$ with boundary $J_{\infty}^{*} \supset J$ and $J_{\infty}^{*}$ has both properties $\mathcal{P}$ and 2 .

4. The necessity of properties $P$ and 2 .

THEOREM I. If there is a homeomorphism of $R^{3}$ onto itself carrying $J$ onto a subset of a plane, then $J$ has property $P$ and property 2.

Proof. Let $h$ be a homeomorphism of a 3 space $\Re$ on $R$ carrying a standard circle $\Im$ on $J$. Let $\Im$ lie in the $x-y$ plane and let $Z$ be the solid sphere in $\Re$ with center in the $x-y$ plane whose boundary meets the $x-y$ plane along $\Im$. Let $\mathfrak{S}=\mathbb{Z} \backslash \mathfrak{\Im}$. Then $\mathfrak{S}$ is homeomorphic to the topological product of a plane $M$ and a closed interval. Taking $S=h(\Im)$ and $K=R \backslash J$, by application of Theorem 7 of [9] a polyhedron $P$ homeomorphic to $M$ is found. Evidently $\mathrm{Cl} P$ is a disk with boundary $J$ and $\mathrm{Cl} P$ is locally polyhedral $\bmod J$.

By a slightly modified argument it may be shown that $J$ also has property $P$.

5. The normalization process for intersections.

5.1. Lemma. Let $G$ and $\Delta$ be disks which are locally polyhedral modulo 
$J=\partial G$ with $J \cap \Delta=a, a$ point, and $\nu(\partial \Delta, J)=1$. Then there is a disk $D$ that, like $\Delta$, is locally polyhedral mod $J$, meets $J$ only at a, and has $\nu(\partial D, J)=1$, and which has the additional property that $D \cap G$ is $l$, an arc from a to $q=G \cap \partial D$.

Proof. For a fixed $\alpha,-1 \leqq \alpha \leqq 1$, let $p_{\alpha}$ denote the point in $R^{3}$ with coordinates $(0,0, \alpha)$, and let $p_{1}, p_{2}, p_{3}$, and $p_{4}$ denote the points $(1,0,0)$, $(0,1,0),(-1,0,0)$, and $(0,-1,0)$ respectively. Then let $A_{\alpha}$ denote the union of the four triangular 2-cells with vertices $p_{\alpha} p_{1} p_{2}, p_{\alpha} p_{2} p_{3}, p_{\alpha} p_{3} p_{4}$, and $p_{\alpha} p_{4} p_{1}$. For each such $\alpha, A_{\alpha}$ is seen to be a disk and any pair $A_{\alpha}, A_{\beta}$ of these disks meet only in their common boundary which is the union of the four segments $p_{1} p_{2}, p_{2} p_{3}, p_{3} p_{4}$, and $p_{4} p_{1}$. The set $B=U_{-1 \leqq \alpha \leqq 1} A_{\alpha}$ is a topological 3-cell.

Now, for each vertex $\sigma_{i}$ of $G \backslash J$ let a line segment $l$ with midpoint $\sigma_{i}$ be chosen. A properly chosen $l_{i}$ meets $G$ only at $\sigma_{i}$ and its end points, $\gamma_{i}$ and $\delta_{i}$, lie on opposite sides of $G$. The length of each $l_{i}$ can be so taken that whenever $\sigma_{i}, \sigma_{j}$, and $\sigma_{k}$ are vertices of a simplex of $G \backslash J$, then the polyhedral solids with vertices $\sigma_{i}, \sigma_{j}, \sigma_{k}, \gamma_{i}, \gamma_{j}, \gamma_{k}$ and $\sigma_{i}, \sigma_{j}, \sigma_{k}, \delta_{i}, \delta_{j}, \delta_{k}$ have only the simplex $\sigma_{i} \sigma_{j} \sigma_{k}$ in common with $G$.

The lengths of the $l_{i}$ can also be taken so that if $\left\{\sigma_{i}\right\}$ is a sequence of vertices of $G \backslash J$ with limit point $\sigma$ in $J$, then the corresponding sequence of lengths has limit zero. If this is done then the union of $J$ and the set of all the polyhedral solids is a 3-cell and hence is $h(B)$ for some homeomorphism $h$ which carries $A_{0}$ onto $G$ and $\partial A_{\alpha}$ onto $J$ for every $\alpha$.

The complexes $G \backslash a$ and $\Delta \backslash a$ are supposed in relative general position (every choice of a simplex from each gives a pair of simplices which are either disjoint or both are Euclidean and their vertices form a set in general position). It may be assumed then, since $G$ contains no vertex of $\Delta \backslash a$, that the lengths of the $l$ were chosen sufficiently small to assure that $h(B)$ contains no vertices of $\Delta \backslash a$ and meets no simplex of $\Delta \backslash a$ except those met by $G$. Thus any simplex of $\Delta \backslash a$ which meets $h(B)$ must meet each $h\left(A_{\alpha}\right)$, and the following notation may be adopted.

$$
\Delta \cap h\left(A_{\alpha}\right)=P_{1}^{\alpha} \cup \ldots \cup P_{k}^{\alpha} \cup P_{k+1}^{\alpha} \cup \ldots \cup P_{m}^{\alpha} \cup \cup_{i} Q_{i}^{\alpha},
$$

where:

$P_{i}^{\alpha}$ is an arc from $a$ to $q_{i}^{\alpha} \in \partial \Delta$ if $i \leqq k$;

$P_{i}^{\alpha}$ is an arc from $q_{i}^{\alpha}$ to $r_{i}^{\alpha}$, both $q_{i}^{\alpha}$ and $r_{i}^{\alpha}$ in $\partial \Delta$, if $k<i \leqq m$;

$P_{i}^{\alpha} \cap P_{j}^{\beta}=\square$ unless both $i$ and $j$ are less than $k+1$, in which case $P_{i}^{\alpha} \cap P_{j}^{\beta}$ $=a(j \neq i)$;

$Q_{i}^{\alpha}$ is a simple closed curve such that $P_{j}^{\beta} \cap Q_{i}^{\alpha}=\square$ or $a$; and

$Q_{i}^{\alpha} \cap Q_{j}^{\beta}$ is either $\square$ or $a(j \neq i)$.

Each curve $Q_{i}^{\alpha}$ bounds an open sub-disk $X_{i}^{\alpha}$ of $h\left(A_{\alpha}\right)$ and the set of all these sub-disks is partially ordered by set inclusion. The choice of the $h(B)$ 
assures that $X_{i}^{0} \subset X_{j}^{0}$ implies $X_{i}^{\alpha} \subset X_{j}^{\alpha}$ for every $\alpha$, and that $X_{i}^{\alpha}$ is disjoint from every $X_{j}^{\beta}$ with $\beta \neq \alpha$, independent of the choice of $i$ and $j$.

It is easily seen that for any $i$ the disk $X_{i}^{0}$ is a subset of only a finite number of disks $X_{i_{1}}^{0}, X_{i_{2}}^{0}, \cdots, X_{i_{k}}^{0}$, and hence determines a unique maximal disk $X_{j(i)}^{0}$ which contains it. It may therefore be assumed that if $i<j$ then either $X_{i}^{0} \supset X_{j}^{0}$ or $X_{1}^{0} \cap X_{j}^{0}=\square$.

Each $Q_{i}^{\alpha}$ also bounds an open sub-disk $Y_{i}^{\alpha}$ of $\Delta$. This set of all $Y_{i}^{\alpha}$ is also partially ordered by set inclusion. It is noted that for a fixed $i$ the set of all $Q_{i}^{\alpha},-1 \leqq \alpha \leqq 1$, is an annular ring or a "pinched" annular ring on $\Delta$ and that one of the following two statements holds: (1) $Y_{i}^{\alpha} \subset Y_{1}^{\beta}$ if and only if $\alpha \leqq \beta$, or (2) $Y_{i}^{\alpha} \subset Y_{i}^{\beta}$ if and only if $\alpha \geqq \beta$. From this fact, a function $\gamma(i)$ can be defined on the positive integers to the reals. Let $\gamma(i)$ be $i /(1+i)$ or $-i /(1+i)$ according as (1) or (2) holds. This function has the following properties, which can be verified from its definition and will be useful later: For $i=1,2, \cdots$,

5.11. $Y_{i}^{0}$ is a proper subset of $Y_{i}^{\gamma(t)}$, so that $Q_{i}^{0} \subset Y_{i}^{\gamma(t)}$,

5.12. $Y_{i}^{\gamma(i)} \supset Q_{i}^{\beta}$ if $|\gamma(i)| \leqq|\beta|$.

As with the $X_{i}^{\alpha}$ it is seen that among the collection of all $Y_{i}^{(i)}$ there are only a finite number which contain any one $Y_{j}^{\gamma(j)}$. Let $S$ be the set of indices $s$ such that $Y_{s}^{\gamma(s)}$ is a sub-disk of no other $Y_{i}^{\gamma(t)}$. Define

$$
D_{1}=\left[\Delta \backslash \bigcup_{s \in S} Y_{s}^{\gamma(s)}\right] \cup \underset{s \in S}{\bigcup} X_{s}^{\gamma(s)}
$$

A map $g$ of $\Delta$ onto $D_{1}$ will now be defined and shown to be a homeomorphism. For each $s$ in $S$ let $g_{s}$ be a homeomorphism of $\mathrm{Cl}\left(Y_{s}^{\gamma(s)}\right)$ onto $\mathrm{Cl}\left(X_{s}^{\gamma(s)}\right)$ which is the identity on their common boundary $Q_{s}^{\gamma(s)}$. Then define $g$ by

$$
g(x)= \begin{cases}x, & x \in\left[\Delta \backslash \bigcup_{s \in S} Y_{s}^{\gamma(s)}\right], \\ g_{s}(x), & x \in Y_{s}^{\gamma(s)}, s \in S .\end{cases}
$$

It is apparent that $g$ is continuous, open, and single-valued. But $g$ is then also 1-1 unless some point $y$ of $D_{1}$ is the image of two points of $\Delta$. If $g(x)$ $=g\left(x^{\prime}\right)=y$ with $x \neq x^{\prime}$, then only one of the points $x, x^{\prime}$ can lie in $\Delta \backslash \bigcup_{s \in S} Y_{s}^{\gamma(s)}$, for $g$ is the identity on this set. So let $x^{\prime}$ be in $Y_{t}^{\gamma(t)}$ for some $t \in S$. Since $g_{s}\left(Y_{s}^{\gamma(s)}\right)=X_{s}^{\gamma(s)}$ is contained in $A_{\gamma(s)}, x$ could not lie on any $Y_{s}^{\gamma(s)}$ and have the same image as $x^{\prime}$, so $x$ lies on $\Delta \backslash \bigcup_{s \in S} Y_{s}$ and $g(x)=x=g_{t}\left(x^{\prime}\right)$.

Thus $x$ lies on $\Delta$ and, as the image of $x^{\prime} \in Y_{s}^{\gamma(s)}$, must also lie on $X_{s}^{\gamma(s)}$ and hence on $A_{\gamma(s)}$. By the notation adopted for $\Delta \bigcap A_{\gamma(s)}$, this requires that $x$ be a point of $Q_{i}^{\gamma(t)}$ for some $i$. This, in turn, requires that $X_{i}^{\gamma(t)}$ be a proper subset of $X_{t}^{\gamma(t)}$, which means the inequality $i>t$ holds. 
But $i>t$ means $i /(1+i)>t /(1+t)$, so $|\gamma(i)|>|\gamma(t)|$. By 5.12 then $Y_{i}^{\gamma(t)} \supset Q_{i}^{\gamma^{(t)}}$, so $x \in Y_{i}^{\gamma^{(t)}}$. But $Y_{i}^{\gamma^{(i)}}$ is a sub-disk of some maximal $Y_{s}^{\gamma^{(s)}}$, so that $x$ cannot be in the set $\Delta \backslash U_{s \in S} Y_{s}^{\gamma(s)}$. This contradiction proves that $g$ is 1-1 and hence a homeomorphism.

Thus $D_{1}$ is a disk, and since no disk $Y_{t}^{\gamma(t)}$ is in $D_{1}, 5.11$ guarantees that $D_{1} \cap G$ does not include any $Q_{i}^{0}$. Since $G \cap \cup_{s \in S} X_{s}^{\gamma(s)}=\square$ (for $X_{s}^{\gamma(s)} \subset\left[A_{\gamma(s)} \backslash J\right]$ and $\gamma(s) \neq 0)$, the relation $D_{1} \cap G \subset \Delta \cap G=P_{1} \cup \ldots \cup P_{m} \cup \cup Q_{i}$ holds. Since each $P_{i}$ is in $\Delta \backslash U_{\bullet \in S} Y_{s}^{\gamma(s)}$ and hence in $D_{1}$,

$$
D_{1} \cap G=P_{1} \cup \ldots \cup P_{k} \cup P_{k+1} \cup \ldots \cup P_{m} .
$$

Now for each $i$ with $k<i \leqq m$, the set $\partial D_{1} \cup P_{i}$ is the boundary of $M_{i} \cup N_{i}$, where $M_{i}$ is an open disk on $D_{1}$ containing $a$ and $N_{1}$ is an open disk on $D_{1}$ not containing $a$. A straightforward argument shows $D_{2}=\bigcap_{i=1}^{m} \mathrm{Cl}\left(M_{i}\right)$ satisfies all the requirements for the disk $D$ except that $D_{2} \cap G=P_{1} \cup \ldots \cup P_{k}$ where each $P_{i}$ is an arc from $a$ to $q_{i}=P_{i} \cap \partial D_{2}$ and $P_{i} \cap P_{j}=a$ for $i \neq j$.

The set $G \backslash \bigcup_{i=1}^{k} P_{i}$ is connected, since the set removed from the disk $G$ is a tree with one point on $\partial G$, so for each pair of indices $i, j$ with $1 \leqq i<j \leqq m$ an $\operatorname{arc} R_{i j}$ can be found on $G \backslash \cup_{i-1}^{k} P_{i}$ joining $q_{i}$ and $q_{j}$. Each set $P_{i} \cup R_{i j} \cup P_{j}$ bounds a sub-disk $X_{i j}$ of $G$, and for some pair of indices, say $u$ and $v$, the relation $X_{u v} \cap D_{2}=P_{u} \cup P_{v}$ holds. Considered as a subset of $D_{2}$, the curve $P_{u} \cup P_{v}$ determines a pair of sub-disks $Y_{u v}$ and $Z_{u v}$ on $D_{2} .\left(X_{u v}, Y_{u v}\right.$, and $Z_{u v}$ are taken as closed disks, and $Y_{u v} \cup Z_{u v}$ is then $D_{2}$.)

The disks $X_{u v}, Y_{u v}$, and $Z_{u v}$ are continuous chains in the mod 2 homology theory. As a chain, $D_{2}=Y_{u v}+Z_{u v}$, so $\partial\left(X_{u v}+Y_{u v}\right)+\partial\left(X_{u v}+Z_{u v}\right)=\partial D_{2}$ must link $J$. But each of the sets $X_{u v} \cup Y_{u v}, X_{u v} \cup Z_{u v}$ is a disk meeting $J$ at a single point, so for a proper choice of notation $\partial\left(X_{u v}+Y_{u v}\right)$ links $J$ (and $\partial\left(X_{u v}+Z_{u v}\right)$ does not $)$. Let $D_{3}=X_{u v} \cup Y_{u v}$.

Then $D_{3}$ is seen to satisfy all the requirements for $D$ except that $D_{3} \cap G=X_{u v} \cup P_{i_{1}} \cup P_{i_{2}} \cup \ldots \cup P_{i_{n}}$, where since $P_{u}$ and $P_{v}$ are in $X_{u_{v}}$ the $n$ can be assumed to be at most $k-2$. There is an obvious semi-linear deformation of $D_{3}$ away from $G$ which is the identity outside an arbitrarily small neighborhood of $X_{u_{v}}$ and such that the image of every point of $X_{u v} \backslash a$ (except possibly those in one of the sets $P_{u}$ or $P_{v}$ ) is in the complement of $G$. The resulting set $D_{4}$ is seen to be a disk satisfying all the requirements for $D$ except that

$$
D_{4} \cap G=P_{j_{1}} \cup P_{j_{2}} \cup \ldots \cup P_{j_{w}},
$$

where $w$ is at most $k-1$.

Repeating this process at most $k-1$ times then produces a disk $D$ as required.

5.2. Lemma. Let $J$ be a simple closed curve with properties $\Phi$ and 2 . Then 
there is a disk $G$ with $\partial G=J$ which is locally polyhedral mod $J$ and a sequence $\left\{D_{i}\right\}_{1}^{\infty}$ of disks with the following properties: For $i=1,2, \cdots$,

$1.1 D_{i} \cap J=a_{i}, a$ point,

$1.2\left\{a_{i}\right\}$ is dense on $J$,

$1.3 D_{i}$ is locally polyhedral modulo $J$,

$1.4 \partial D_{i}$ links $J$,

$1.5 D: C S\left(a_{i}, 1 / i\right)$,

$1.6 D_{i} \cap D_{j}=\square$ when $i \neq j$,

$1.7 D_{i} \cap G=l_{i}$, an arc.

Now, since $J$ is separable, it contains a countable dense subset $\left\{c_{i}\right\}$. Since $J$ has property $P$, a collection $\left\{K_{i n}\right\}$ of spheres can be found such that $K_{i n}$ is locally polyhedral at each of its points except the two unique points $a_{i n}$ and $b_{i n}$ of $K_{i n} \cap J$, such that $K_{i n}$ contains $c_{i n}$ in its interior, and such that $K_{i n} \subset S\left(c_{i}, 1 / n\right), i, n=1,2, \cdots$. Choose a sub-disk $\Delta_{i n}$ of each $K_{\text {in }}$ such that $\Delta_{i n} \cap J=a_{i n}$, and let $\left\{\Delta_{p}\right\}$ be an ordering of this sequence of disks. This sequence evidently satisfies 1.1 and since $\lim _{n \rightarrow \infty} a_{i n}=c_{i}$, also satisfies 1.2. Condition 1.3 for any $\Delta_{p}$ follows since it is a part of some $K_{\text {in }}$ which is locally polyhedral except at $a_{i n}$ and $b_{i n}^{\prime}$, and since $\Delta_{p}$ does not contain $b_{i n}^{\prime}$. Topologically, $\Delta_{p}$ is the northern hemisphere and $\partial \Delta_{p}$ the equator of the corresponding sphere $K_{i n}$, while $J$ consists of a pair of arcs joining $a_{i n}$ and $b_{i n}$, one interior and one exterior to $K_{i n}$. This makes it evident that $\partial \Delta_{p}$ links $J$ so 1.4 holds.

For each $i$, Lemma 5.1 can be applied to $G$ and $\Delta_{i}$ to yield a new disk $D_{i}$ which satisfies 1.7 as well as 1.1 through 1.4 . Suppose now that $\left\{D_{i}\right\}$ is any sequence of disks satisfying 1.1 and any one of the other conditions in the list, say $m$. If for each $i$ a sub-disk $D_{i}^{\prime}$ of $D_{i}$ is chosen so that $a_{i} \in D_{i}^{\prime} \backslash \partial D_{i}^{\prime}$ (and $\partial D_{i}^{\prime} \cap l$ is a point, if $m=1.7$ ), then $\left\{D_{i}^{\prime}\right\}$ also satisfies 1.1 and $m$.

Hence $D_{1}$ may be replaced by a new $D_{1}$ which lies in $S\left(a_{1}, 1\right)$ and has properties $1.1,1.2,1.3,1.4$, and 1.7 . Similarly, $D_{2}$ may be replaced by a sub-disk of itself (and again called $\left.D_{2}\right)$ which lies in $S\left(a_{2}, 1 / 2\right)$, does not meet $D_{1}$, and has all the other properties required of $D_{2}$, and so on.

5.3. Lemma. If $J$ is a simple closed curve with properties $\mathbb{P}$ and 2 , and $\left\{D_{i}\right\}$ is a properly chosen sequence of disks as required by Lemma 5.2, then there is a sequence $\left\{T_{i}\right\}$ of polyhedral tori such that for $i=1,2, \cdots$,

$1.8 J \subset$ Int $T_{i}$,

$1.9 T_{i} \subset S(J, 1 / i) \cap \operatorname{Int} T_{i-1}$,

$1.10 \partial D_{i} \subset\left[\left(\operatorname{Ext} T_{i}\right) \cap\left(\operatorname{Int} T_{i-1}\right)\right]$,

$1.11 D_{i} \cap T_{j}=\left\{\begin{array}{l}r_{i j}, \text { a simple closed curve, } j \geqq i, \\ \square, j<i .\end{array}\right.$

Proof. Let $\left\{D_{i}\right\}$ be the sequence of disks guaranteed to satisfy 1.1 through 1.7 of Lemma 5.2 , and make the assumption that $D_{1}, \cdots, D_{n-1}$ have been 
properly altered and $T_{0}, T_{1}, \cdots, T_{n-1}$ chosen so that $1.8,1.9,1.10$ and 1.11 hold for these sets. On each of the disks $D_{i}, i=1,2, \cdots, n$, choose now a fixed sub-disk $D_{i}^{n}$ having $a_{i}$ as an interior point, lying entirely in $S(J, 1 / 2 n)$, and such that $\partial D_{i}^{n}$ meets $l_{i}$ in a single point. For each $i$ let $\gamma_{i}$ denote $d\left(J, D_{i} \backslash D_{i}^{n}\right)$. Then take $\epsilon_{n}=\operatorname{Min}\left[d\left(J, T_{n-1}\right), d\left(J, \partial D_{n}\right), \gamma_{1}, \cdots, \gamma_{n}, 1 / 2 n\right]$. Then by [8], since $J$ has property $\mathcal{P}$ there is a polyhedral torus $T_{n}$ containing $J$ in its interior and such that $T_{n} \subset S\left(J, \epsilon_{n}\right)$. This states that $T_{n}$ satisfies 1.8 and 1.9, and since it may be supposed that the disk $D_{n}$ was taken to lie entirely in $T_{n-1}$, also guarantees 1.10. The inductive hypothesis together with $D_{n} C$ Int $T_{n-1}$ requires that 1.11 be satisfied for $j<n$ and all $i=1,2, \cdots, n$. Hence only the sets $D_{i} \cap T_{n}$ for $1 \leqq i \leqq n$ need be considered to prove the choice of $T_{n}$ can be made as required.

It will be convenient to suppose a disk $D_{0}$ has been chosen such that $D_{0} \cap J=a_{0}$, a point, $\partial D_{0}$ links $J, D_{0}$ is locally polyhedral mod $J, D_{0} \cap D_{i}=\square$ for $i=1,2, \cdots$, and $D_{0} \cap G=l_{0}$, an arc. It is clear that no simple closed curve on any $D_{i}$ can link $\partial D_{0}$, and the torus $T_{0}$ can be chosen so that $\partial D_{0}$ $C$ Ext $T_{0}$. If this is done, then $J$ and $\partial D_{0}$ generate the 1-dimensional homology group of the complement of $T_{n}$ (for any $n$ ) so that every simple closed curve on $T_{n}$ must bound on $T_{n}$, or link at least one of the curves $J, \partial D_{0}$.

For any fixed $i(1 \leqq i \leqq n), \partial D_{i}$ is a curve in Ext $T_{n}$ and $D_{i} \cap T_{n}$ is the union of a finite collection $r_{i 1}^{\prime}, r_{i 2}^{\prime}, \cdots, r_{i p_{i}}^{\prime}$ of mutually disjoint curves. Since every simple closed curve on $T_{n}$ must link either $J, \partial D_{0}$, both, or neither, the curves $r_{i j}^{\prime}$ are divided in to two types

Type 1: $r_{i j}^{\prime}$ links neither $J$ nor $\partial D_{0}$,

Type 2: $r_{i j}^{\prime}$ links $J$ but not $\partial D_{0}$.

Each $r_{i j}^{\prime}$ bounds a sub-disk $Y_{i j}$ of $D_{i}$ and evidently $Y_{i j}$ does not contain $a_{i}$ or does contain $a_{i}$ according as $r_{i j}^{\prime}$ is of type 1 or of type 2 . Any $r_{i j}^{\prime}$ of type 1 must bound a sub-disk $X_{i j}$ of $T_{n}$, as may readily be seen by looking upon $T_{n} \backslash D_{0}$ as an infinite cylinder which meets the bounded disk $D_{i}$ in the set $T_{n} \cap D_{i}$. As $j$ ranges over the set of integers for which $r_{i j}^{\prime}$ is of type 1 , an index $k$ is found such that $Y_{i k}$ meets $T_{n}$ only at points of $r_{i k}^{\prime}$, and if $T_{n}$ is replaced by the result of deforming $\left(T_{n} \backslash X_{i k}\right) \cup Y_{i k}$ semi-linearly away from $D_{i}$ in a neighborhood of $Y_{i k}$, the number of components $r_{i j}^{\prime}$ of type 1 is reduced. Hence eventually $T_{n} \cap D_{i}$ is a collection $r_{i 1}^{\prime}, \cdots, r_{r_{i}}^{\prime}$ of curves of type 2 and the notation is arranged so that $Y_{i 1} \subset Y_{i 2} \subset \cdots \subset Y_{i r_{i}}$. The pair $r_{11}^{\prime}, r_{i 2}^{\prime}$ bound a unique annular ring $R^{*}$ on $T_{n} \backslash D_{0}$, and an annular ring $R^{\sharp}$ on $D_{i}$ which meets $T_{n}$ in the set $r_{i 1}^{\prime} \cup r_{i 2}^{\prime}$. Thus $\left(T_{n} \backslash R^{*}\right) \cup R^{\sharp}$ is again a polyhedral torus $T^{*}$ and $T_{n}$ is to be replaced by the result of deforming $T^{*}$ semi-linearly in such a way that the component $R^{*}$ of $T^{*} \cap D_{i}$ is reduced to the set $r_{i 2}^{\prime}$. Repetition of this process yields a $T_{n}$ such that $T_{n} \cap D_{i}$ is a single curve $r_{i n}$.

From the fact that the $r_{i j}$ above lie on the old $T_{n}$ and hence interior to $S\left[J, d\left(D_{i} \backslash D_{i}^{n}, J\right)\right]$, it is seen that each of the sets $Y_{i j}$ and the sets $R^{f}$ lie on some $D_{i}^{n}$ and hence in $S(J, 1 / 2 n)$. Consequently, if $p$ is a point of the new 
$T_{n}$, then $p$ is the image under a sequence of deformations of a point $p^{\prime}$, and $p^{\prime}$ was either on the old $T_{n}$ or on one of the $Y_{i j}$ or $R^{f}$, so that $p^{\prime} \in S(J, 1 / 2 n)$. Thus, since it may be assumed that the $k$ th deformation moved no point more than a distance of $1 / 2 k \cdot 1 / 2 n$, the point $p$ must lie in $S(J, 1 / n)$. By restricting the distance any point is moved by the $k$ th deformation still further if necessary, it is clear that the new $T_{n}$ can be made to satisfy conditions $1.8,1.9$, and 1.10 .

This process is repeated for $i=1,2,3, \cdots, n$ in turn, and as a consequence 1.11 is established for $j=n$ and $i \leqq n$. Taking $D_{i}$ to lie in Int $T_{n}$ for all $i>n$ is clearly possible, so 1.11 is established. This proves the lemma.

\section{The normalization theorem.}

Theorem II. Let $J$ be a simple closed curve with properties $\mathbb{P}$ and 2. Then there is a disk $G$ with $\partial G=J$ which is locally polyhedral mod $J$, a sequence $\left\{D_{i}\right\}_{1}^{\infty}$ of disks, and a sequence $\left\{T_{i}\right\}_{0}^{\infty}$ of polyhedral tori having the following properties: For $i=1,2, \cdots$,

$1.1 D_{i} \cap J=a_{i}, a$ point,

$1.2\left\{a_{i}\right\}_{1}^{\infty}$ is dense on $J$,

$1.3 D_{i}$ is locally polyhedral mod $J$,

$1.4 D_{i}$ meets $J$ essentially $\left(\partial D_{i}\right.$ links $\left.J\right)$,

$1.5 D_{i} \subset S\left(a_{i}, 1 / i\right)$,

$1.6 D_{i} \cap D_{j}=\square$ when $j \neq i$,

$1.7 D_{i} \cap G=l_{i}$, an arc,

$1.8 J \subset$ Int $T_{i}$,

$1.9 T_{i} \subset S(J, 1 / i) \cap \operatorname{Int} T_{i-1}$,

$1.10 \partial D_{i} \subset\left[\left(\right.\right.$ Ext $\left.T_{i}\right) \cap\left(\right.$ Int $\left.\left.T_{i-1}\right)\right]$,

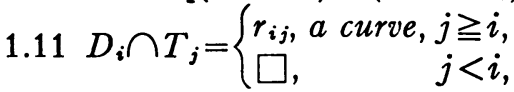

$1.12 G \cap T_{i}=s_{i}$, a curve, and $r_{m i} \cap s_{i}=c_{m i}, a$ point, $m \leqq i$.

Proof. Let $G,\left\{D_{i}\right\}$, and $\left\{T_{i}\right\}$ be the sets guaranteed by Lemma 5.1 and Lemma 5.2 for the curve $J$. These sets meet all of the requirements of the theorem except possibly 1.12 , and it is to be established inductively that by a sequence of alterations of these sets, 1.12 can be made to hold also. Suppose then that $G,\left\{D_{i}\right\}$, and $\left\{T_{i}\right\}$ satisfy all of the requirements of the theorem except 1.12, and that 1.12 holds for $i=1,2, \cdots, n-1$. (Note that this is the case for $n=1$.) Let $m$ be one of the integers $1,2, \cdots, n-1$. Then the set $D_{m} \cap T_{n}=r_{m n}$ is a simple closed curve separating $a_{m}$ from $\partial D_{m}$ on $D_{m}$, since $a_{m} \in J \subset$ Int $T_{n}$ and $\partial D_{m} \subset$ Ext $T_{n}$. The set $G \cap D_{m}=l_{m}$ is an arc from $a_{m}$ to $q_{m} \in \partial D_{m}$, so $r_{m n} \cap l_{m}$ is a finite collection of points $p_{1}, p_{2}, \cdots, p_{k}$, indexed in the order in which they are encountered when $l_{m}$ is traversed from $a_{m}$ to $q_{m}$. Let $A_{0}$ be the closed sub-arc of $l_{m}$ from $a_{n}$ to $p_{1}$, let $A_{i}$ be the closed sub-arc of $l_{m}$ from $p_{i}$ to $p_{i+1}$ for $i=1,2, \cdots, k-1$, and $A_{k}$ be the closed subarc of $l_{m}$ from $p_{k}$ to $q_{m}$. Each pair $p_{i}, p_{i+1}$ divides $r_{m n}$ into a pair of closed arcs 
$B_{i}$ and $C_{i}$ where if $N(S)$ denotes the number of points $p_{i}$ in the set $S$ then $N\left(B_{i}\right)<N\left(C_{i}\right)$. To see that this inequality is always sharp, note that $p_{i}$ and $p_{i+1}$ lie in both $B_{i}$ and $C_{i}$ while $p_{r}$ lies in one but not the other whenever $i \neq r$. Hence $N\left(B_{i}\right)+N\left(C_{i}\right)=k+2$ which is odd since $k$ must be, so $N\left(B_{i}\right) \neq N\left(C_{i}\right)$.

If $k=1$, then $r_{m n} \cap T_{n}=p_{1}$, a point as desired. It must be shown that if $k>1$, then there is a new $T_{n}$ having the properties required of the old and for which $k=1$ can be found. The deformation of the old $T_{n}$ to form the new one can be carried out only after an index $w$ such that $N\left(B_{w}\right)=2$ is found, so the next few paragraphs are concerned with showing such an index exists.

For any index $i$ the triple $A_{i}, B_{i}, C_{i}$ is a triple of arcs from $p_{i}$ to $p_{i+1}$ which are mutually disjoint except for end points. Letting $A_{i}^{0}, B_{i}^{0}, C_{i}^{0}$ denote the open arc obtained by removing the end points from $A_{i}, B_{i}, C_{i}$ respectively, this requires that, for each $i, A_{i} \cup B_{i}$ be a simple closed curve on $D_{m}$ and that $C_{i}^{0}$ lie either in the interior or the exterior of $A_{i} \cup B_{i}$ (relative to $D_{m}$ ). It will first be shown that for some index $v, C_{v}^{0}$ is exterior to $A_{v} \cup B_{v}$.

For suppose $C_{i}^{0}$ is interior to $A_{i} \cup B_{i}$ for every $i$. Then, choosing an arbitrary $x, N\left(C_{x}\right)+N\left(B_{x}\right)=k+2$ and $N\left(C_{x}\right)>N\left(B_{x}\right)$. So $2 N\left(C_{x}\right)>k+2$ and since $k$ is odd and $N\left(C_{x}\right)$ is an integer, $N\left(C_{x}\right) \geqq(k+3) / 2$. A straightforward argument shows that for any odd integer $k$ and subset $S$ of the integers $1,2, \cdots, k$ with cardinal greater than or equal to $(k+3) / 2$, there are at least two distinct integers $p$ and $q$ such that $p, q, p+1$, and $q+1$ are all in $S$. Applying this to the set of indices $\left\{j \mid p_{j}\right.$ lies on $\left.C_{x}\right\}$, an index $y \neq x$ is found such that $p_{y}$ and $p_{y+1}$ both lie on $C_{x}$. This means that either $p_{y}$ or $P_{y+1}$ is a point of $C_{x}^{0}$ and hence is interior to $A_{x} \cup B_{x}$. Since $A_{y}$ can meet $A_{x} \cup B_{x}$ in at most one end point, it follows that $A_{y}^{0}$ is interior to $A_{x} \cup B_{x}$. If in addition $B_{y} \subset C_{x}$, then $A_{y} \cup B_{y}$ is interior to $A_{x} \cup B_{x}$ except possibly for a single point, so that $B_{x}^{0}$ is exterior to $A_{y} \cup B_{y}$. But, taking complements relative to $r_{m n}$, $B_{y} \subset C_{x}$ also implies $B_{x} \subset C_{y}$ so $C_{y}$ is exterior to $A_{y} \cup B_{y}$, contradicting our assumption.

Hence, since $p_{y}$ and $p_{y+1}$ are points of $C_{x}$ and $B_{x} \subset C_{y}$ is false, the relation $C_{y} \subset C_{x}$ must hold, so that $N\left(C_{y}\right) \leqq N\left(C_{x}\right)$. But this inequality must be sharp, since either $p_{x}$ or $p_{x+1}$ is on $C_{x}$ but not on $C_{y}$.

Summarizing, if for each $i$ the arc $C_{i}^{0}$ is interior to $A_{i} \cup B_{j}$, then for each index $x$ there is an index $y$ such that $N\left(C_{y}\right)<N\left(C_{x}\right)$. This impossibility establishes the existence of an index $v$ such that $C_{0}^{0}$ is exterior to $A_{v} \cup B_{v}$.

Now if $N\left(B_{v}\right)>2$ for the $v$ just found, then $B_{v}$ meets $l_{m}$ at some point $p_{i}$ with $v \neq i \neq v+1$. But then $A_{j}^{0}$ is interior $A_{v} \cup B_{v}$ for $j=i$ or $j=i-1$, since each point of contact of $l$ with $r_{m n}$ is a crossing point of these curves by the relative general position requirement. Since $A_{j}^{0}$ is interior and $C_{0}^{0}$ is exterior to $A_{v} \cup B_{v}$, both end points of $A_{j}$ lie on $B_{v}$. Thus $B_{j}$ is a proper subset of $B_{v}$ and $N\left(B_{v}\right)>N\left(B_{j}\right)$. Further, $C_{j}^{0} \supset C_{0}^{0}$ so that $C_{j}^{0}$ must be exterior to $A_{j} \cup B_{j}$ and repetition is possible if $N\left(B_{j}\right)>2$.

That is to say, a sequence of indices $v, v_{1}, v_{2}, \ldots$ can be found such that 
$N\left(B_{v}\right)>N\left(B_{v_{1}}\right)>N\left(B_{v_{2}}\right)>\cdots$ and this sequence cannot terminate unless $N\left(B_{v_{k}}\right)=2$ for some $k$. Since only a finite number of indices are possible, there is a $w$ such that $N\left(B_{w}\right)=2$.

The simple closed curve $A_{w} \cup B_{w}$ bounds a sub-disk $D_{m w}$ of $D_{m}$ and $D_{m w} \cap G=A_{w}$ while $D_{m w} \cap T_{n}=B_{w}$. The disk $D_{m w}$ can be inflated to form a polyhedral sphere $S_{m v}$ which meets $D_{m}$ in $A_{w} \cup B_{w}$, and $T_{n}$ in $B_{w}$. If $S_{m v}$ and $T_{n}$ are each slit along $B_{w}$ and the corresponding edges joined, the resulting set is a new $T_{n}$ whose intersection with $D_{i}$ is unchanged unless $i=m$ and whose intersection with $D_{m}$ is $\left(r_{m n} \backslash B_{w}\right) \cup A_{w}$.

The general position requirements make it necessary that if $A_{w}$ is interior (exterior) to the old $T_{n}$, then $A_{w-1}$ and $A_{w+1}$ are both exterior (interior) to the old $T_{n}$. Since $D_{m w}$ meets $l_{m}$ only in $A_{w}$, then $A_{w}$ lies on the new $T_{n}$ while $A_{w-1}$ and $A_{w+1}$ are both interior or both exterior to it. Thus a slight deformation of the new $T_{n}$ away from $l_{m}$ in a neighborhood of $A_{w}$ makes the set $A_{w-1}$ $\cup A_{w} \cup A_{w+1}$ disjoint from the new $T_{n}$ except for the points $p_{w-1}$ and $p_{w+2}$.

Thus the intersection of the new $T_{n}$ with $D_{m} \cap G$ is exactly $p_{1}, \cdots, p_{w-1}$, $p_{w+2}, \cdots, p_{k}$, i.e., the number of points in $T_{n} \cap D_{m} \cap G$ has been reduced by two. Since this process can be repeated whenever $k>1$ and since $k$ is always odd, eventually a $T_{n}$ is found such that $k=1$ so that $r_{m n} \cap l_{m}=D_{m} \cap T_{n} \cap G$ $=C_{m n}$, a point.

Each deformation takes place in a neighborhood of $D_{m}$ which can be taken so small that no $D_{i}$ other than $D_{m}$ meets it, so the new $T_{n}$ has the same intersection with the other $D_{i}$ as the old.

An argument similar to that used in Lemma 2 shows that the new $T_{n}$ lies in $S(J, 1 / n)$ provided the old one was taken in $S\left(J, \epsilon_{n}\right)$ for sufficiently small $\epsilon_{n}$. To outline this argument briefly, a sub-disk $D_{m}^{n}$ of $D_{m}$ is chosen so as to contain $a_{m}$ and lie in $S(J, 1 / 2 n)$. Then $\epsilon_{n}$ is taken sufficiently small to guarantee that any sub-disk of $D_{m}$ bounded by a subset of $r_{m n} \cup l$ is a subset of $D_{m}^{n}$. Thus any point of the new $T_{n}$ came from a point of $S(J, 1 / 2 n)$ by a sequence of deformations which can be taken so small that the final image is in $S(J, 1 / n)$. Similarly the remainder of the conditions $1.8,1.9$, and 1.10 can be made valid for the new $T_{n}$. Thus after the process is applied for $D_{m}$ the sets $T_{1}, T_{2}, \cdots, T_{n-1}$, the new $T_{n},\left\{D_{i}\right\}$, and the remainder of the $T_{i}$ form a collection which satisfy the requirements of the theorem except possibly for 1.12 , and $T_{n} \cap G \cap D_{m}=C_{m n}$, a point.

Suppose then that this process has been performed for $m=1,2, \cdots, n$ in succession, and consider the intersection of the final $T_{n}$ with $G$. Relative general position may be assumed, so $T_{n} \cap G$ is the union of a finite collection of mutually disjoint simple closed curves $s_{1 n}, s_{2 n}, \cdots, s_{t n}$. Since for $k=1,2, \cdots$, and $n, r_{k n} \cap l_{k}=\left(D_{k} \cap T_{n}\right) \cap\left(D_{k} \cap G\right)=C_{k n}$, a point, it follows that $D_{k}$ meets but one of the curves $s_{i n}$. Suppose the notation is chosen so that $D_{1} \cap\left(T_{n} \cap G\right)$ is a point of $s_{1 n}$. Then $s_{1 n}$ separates $q_{1}$ and $J=\partial G$ on $G$.

Now let $A_{k}$ be an arc in $G \backslash J$ from $q_{1}$ to $q_{k}, k=1,2, \ldots$. It can be sup- 
posed that in addition to the other requirements on the $\epsilon_{n}$ of Lemma 2 [where $T_{n}$ was chosen to lie in $S\left(J, \epsilon_{n}\right)$ ] that $\epsilon_{n}$ was small enough to guarantee that $A_{1} \cup A_{2} \cup \ldots \cup A_{n}$ be exterior to $T_{n}$. But then $A_{k} \cup l_{k}$ contains an arc from $q_{1}$ to $a_{k} \in J$, and hence meets $s_{1 n}$. Since $s_{1 n}$ is on $T_{n}$ and cannot meet $A_{k}$, $s_{1 n}$ and $l_{k}$ must meet, so the disk $D_{k}$ meets $s_{1 n}$. As a consequence, each of the disks $D_{1}, D_{2}, \cdots, D_{n}$ meets $s_{1 n}$ and does not meet any other component of $T_{n} \cap G$.

Thus each $s_{j n}, j \neq 1$, bounds a sub-disk $F_{j}$ of $G$. Since $\partial F_{j} \cap D_{i}=s_{j_{n}} \cap D_{i}=\square$ for each $i$, if $F_{j}$ meets any $D_{i}$ it must do so in a collection of simple closed curves. But $F_{j} \cap D_{i} \subset G \cap D_{i}=l_{i}$, an arc, so this is impossible and the conclusion $F_{j} \cap D_{i}=\square$ is reached.

This means that $s_{j n}$ does not link any $\partial D_{i}$, and $s_{j n}$ cannot link $J$, the boundary of a disk containing it. Therefore $s_{j n}$ also bounds a disk $E_{j}$ on $T_{n}$, and $s_{1 n} \subset E_{j}$ leads to a contradiction, since $s_{1 n}$ links each $\partial D_{i}$.

Summarizing, for $j=2,3, \cdots, t, s_{j n}$ bounds a pair of disks $E_{j}$ on $T_{n}$ and $F_{j}$ on $G$, each of which is disjoint from $s_{1 n}$ and consequently $D_{1} \cup D_{2} \cup \ldots$ $\cup D_{n}$. For some $j$ the disk $F_{j}$ meets $T_{n}$ only in $s_{j n}=\partial F_{j}=\partial E_{j}$. Following the now familiar process, $T_{n}$ is replaced by the result of deforming $\left(T_{n} \backslash E_{j}\right) \cup F_{j}$ away from $G$ semi-linearly in a neighborhood of $F_{j}$, and the new $T_{n}$ has fewer components of intersection with $G$. A finite number of repetitions yields a $T_{n}$ such that $T_{n} \cap G=s_{n}$ (the old $s_{1 n}$ ) as required. The argument to show $T_{n} \subset S(J, 1 / n)$ can be repeated here, and this establishes the theorem.

7. Concentric toral theorem. In this section we have occasion to refer to the boundary of certain chains on polyhedral complexes. Except in 7.9 these may be taken modulo 2. For 3-dimensional chains in $R$ these boundaries may be identified with point-set boundaries.

The following theorem asserts, essentially, that a pair of suitably defined "concentric" polyhedral tori bound a region whose closure is homeomorphic to the topological product of a torus and a closed interval.

Theorem III. Let $T_{1}$ and $T_{2}$ be polyhedral tori in $R$ subject to the conditions:

(a) $T_{2}$ lies in the interior of $T_{1}$;

(b) there is a disk $G$ such that

$\left(\beta_{1}\right) \partial G=J$ is interior to $T_{2}$;

$\left(\beta_{2}\right) G \cap T_{i}$ is a single curve $s_{i} ; i=1,2$;

$\left(\beta_{3}\right) G$ is polyhedral mod $J$;

(c) there is a pair of disks $D^{a}, D^{b}$ such that

$\left(\gamma_{1}\right) D^{a} \cap D^{b}=\square$;

$\left(\gamma_{2}\right) D^{a} \cap J=a, D^{b} \cap J=b$, where $a, b$ are points;

$\left(\gamma_{3}\right) D^{a}\left(D^{b}\right)$ is polyhedral mod $J$;

$\left(\gamma_{4}\right)$ if we define $D_{i}^{a}=D^{a} \cap\left(\mathrm{Cl}\right.$ Int $\left.T_{i}\right)$, and similarly for $D_{i}^{b}$, then $r_{i}^{a}$ $=\partial D_{i}^{a}, r_{i}^{b}=\partial D_{i}^{b}$ is a single curve;

$\left(\gamma_{5}\right) r_{i}^{a}, r_{i}^{b}$ and $s_{i}$, regarded as 1-cycles, satisfy $r_{i}^{a} \times s_{i}, r_{i}^{b} \times s_{i}, r_{i}^{a} \times 0$, $r_{i}^{b} \times 0, s_{i} \times 0$ on $T_{i} ; i=1,2$; 
(d) $D_{i}^{a} \cap G\left(D_{i}^{b} \cap G\right)$ is an arc

then $U=\left(\operatorname{Int} T_{1}\right) \cap\left(\right.$ Ext $\left.T_{2}\right)$ has a closure homeomorphic to $T_{1} \times[0,1]$ or $T_{2} \times[0,1]$.

7.1. This paragraph is devoted to showing that the hypotheses of Theorem III are fulfilled as a consequence of the constructions in Theorem II. Using the notations of the latter, condition (a) follows from the existence of the polyhedral tori asserted in Theorem II and 1.9. Part $\left(\beta_{1}\right)$ of (b) follows from the existence of $G$ (property $Q$ ) and 1.8. Part $\left(\beta_{2}\right)$ is a consequence of $1.12,\left(\beta_{3}\right)$ from the existence of $G$. Parts $\left(\gamma_{1}\right),\left(\gamma_{2}\right),\left(\gamma_{3}\right)$, and $\left(\gamma_{4}\right)$ follow from 1.6, 1.1, 1.1 and 1.3, and 1.11, respectively. To see $\left(\gamma_{5}\right)$ is fulfilled we note first $r_{i}^{a}$ links $J$ by 1.4. Since $J \cap T_{i}=\square, r_{i}^{a} \times 0$ on $T_{i}$ and similarly for $r_{i}^{b}$. To see $s_{i} \times 0$ on $T_{i}$ first observe $s_{i}$ links $\partial D_{0}$, where $D_{0}$ is defined in the second paragraph of the proof of Lemma 5.3, for, if we suppose $s_{i} \sim 0$ on $R \backslash \partial D_{0}$, then $J \sim 0$ on $R \backslash \partial D_{0}$, which is impossible. Since $\partial D_{0} \cap T_{i}=\square$, hence $s_{i} \nsim 0$ on $T_{i}$. Next, if $r_{i}^{a} \sim s_{i}$ on $T_{i}$, then since $s_{i}$ links $\partial D_{0}, r_{i}^{a}$ links $\partial D_{0}$, which is a contradiction. Finally, (d) follows from 1.7.

7.2. Preliminaries to the proof of Theorem III. The curves $r_{i}^{a}, r_{i}^{b}$, and $s_{i}$ defined in $\left(\gamma_{4}\right)$ and $\left(\beta_{2}\right)$ may be regarded as 1 -cycles on $T_{i}$. By $\left(\gamma_{5}\right), s_{i} \times 0$ on $T_{i}$. Similarly, $p_{i}^{a} \times 0, p_{i}^{b} \times 0$ on $T_{i}$. To put it differently, no one of the curves $s_{i}, r_{i}^{a}$, or $r_{i}^{b}$ separates $T_{i}$. Since the 1-dimensional homology group of $T_{i}$, $H^{1}\left(T_{i}\right)$, has rank two, however, there is a homology connecting $s_{i}, r_{i}^{a}$, and $r_{i}^{b}$. By $\left(\gamma_{1}\right)$ and $\left(\gamma_{4}\right), r_{i}^{a}$ and $r_{i}^{b}$ as sets are disjoint. Since neither $r_{i}^{a}$ nor $r_{i}^{b}$ bounds and $s_{i}$ meets $r_{i}^{b}$ in a single point (Theorem II, 1.12), a glance at the universal covering complex of $T_{i}$ shows that the homology relation between $s_{i}, r_{i}^{a}$, and $r_{i}^{b}$ is in fact a relation between $r_{i}^{a}$ and $r_{i}^{b}$. That is to say, $r_{i}^{a}$ and $r_{i}^{b}$ together do cut $T_{i}$.

Let the components of $T_{i} \backslash\left(r_{i}^{a} \cup r_{i}^{b}\right)$ be $C_{i}^{\prime}$ and $C_{i}{ }^{\prime}, i=1,2$; then $C_{i}^{\prime} \cap\left\{D_{i}^{a} \cup D_{i}^{b}\right\}=\square$. Then $K_{i}^{\prime}=C_{i}^{\prime} \cup D_{i}^{a} \cup D_{i}^{b}$ consists of a pair of disjoint disks, each meeting a circular ring only along its boundary, and in such a way that $K_{i}^{\prime}$ is a topological 2 -sphere. Similarly, $K_{i}^{\prime \prime}=C_{i}{ }^{\prime} \cup D_{i}^{a} \cup D_{i}^{b}$ is a topological 2-sphere. The notation is adjusted so that $C_{2}^{\prime \prime}$ lies interior to $K_{1}^{\prime \prime}$ rather than exterior.

Define $Q^{\prime}=\mathrm{Cl}\left\{\left(\operatorname{Int} K_{1}^{\prime}\right) \cap\left(\operatorname{Ext} K_{2}^{\prime}\right)\right\}$ and $Q^{\prime \prime}=\mathrm{Cl}\left\{\left(\operatorname{Int} K_{1}^{\prime \prime}\right) \cap\left(\operatorname{Ext} K_{2}^{\prime \prime}\right)\right\}$. Inspection shows that $\partial Q^{\prime}=C_{1}^{\prime} \cup C_{2}^{\prime} \cup \mathrm{Cl}\left\{D_{1}^{a} \backslash D_{2}^{a}\right\} \cup \mathrm{Cl}\left\{D_{1}^{b} \backslash D_{2}^{b}\right\}$ with a similar formula holding for $\partial Q^{\prime \prime}$. Thus $\partial Q^{\prime}$ and $\partial Q^{\prime \prime}$ are tori. By the fact that $T_{1}$ and $T_{2}$ are polyhedral and $\left(\gamma_{3}\right), \partial Q^{\prime}$ and $\partial Q^{\prime \prime}$ are polyhedral tori.

The set $\partial Q^{\prime}$ contains $r_{2}^{a}$. The disk $D_{2}^{a}$ (see $\left.\left(\gamma_{4}\right)\right)$ is disjoint with $Q^{\prime}$ except for $r_{2}^{a}$. Since $D_{2}^{a}$ is polyhedral save for one point $\left(\gamma_{2}\right)$, namely, where it meets $J, D_{2}^{a}$ may be replaced by a strictly polyhedral disk $E_{2}^{a}$ whose boundary is $r_{2}^{a}$ and which is otherwise entirely exterior to $\partial Q^{\prime}$. (Lemma 1 of HarroldMoise [7] gives this replacement.) Thus $r_{2}^{a}$ is an "unknotted" polyhedral curve in $R$.

The set $\partial Q^{\prime}$ also contains a curve $w^{\prime}$ that is the boundary of a polyhedral 
disk $G^{\prime}$, where $G^{\prime} \backslash w^{\prime}$ is in the interior of $\partial Q^{\prime}$. We define

$$
w^{\prime}=\left(s_{2} \cap K_{2}^{\prime}\right) \cup\left(s_{1} \cap K_{1}^{\prime}\right) \cup\left\{\left(D_{1}^{a} \backslash D_{2}^{a}\right) \cap G\right\} \cup\left\{\left(D_{1}^{b} \backslash D_{2}^{b}\right) \cap G\right\} .
$$

By $\left(\beta_{2}\right),\left(\gamma_{5}\right)$, and the general position requirements, $s_{1} \cap D^{a}=T_{1} \cap G \cap D^{a}$ is a single point and similarly for $s_{1} \cap D^{b}$. An inspection of the definitions of $K_{1}^{\prime}$ and $K_{2}^{\prime \prime}$ now shows that each of $s_{2} \cap K_{2}^{\prime}$ and $s_{1} \cap K_{1}^{\prime}$ are arcs. By (d), $\left(D_{1}^{a} \backslash D_{2}^{a}\right) \cap G$ is an arc and similarly for the last term of $w^{\prime}$ displayed in braces. By rearranging the order of the terms above, we see $w^{\prime}$ is a simple closed curve, in fact, a polyhedral one.

By (a) and $\left(\beta_{1}\right), G \cap\left(\operatorname{Int} T_{1}\right) \cap\left(\right.$ Ext $\left.T_{2}\right)$ is a circular ring. By the preceding paragraph, this ring is divided into two pieces by $D^{a} \cap G$ and $D^{b} \cap G$. From the definitions of $K_{1}^{\prime}$ and $K_{2}^{\prime}$, one of these pieces, say $G^{\prime}$, is bounded by $w^{\prime}$. (A similar calculation holds for the other component $G^{\prime \prime}$ of the complement of the ring relative to $D^{a} \cup D^{b}$, the boundary of $G^{\prime \prime}$ being $w^{\prime \prime}$, where $w^{\prime \prime}$ is defined as $w^{\prime}$ is above, replacing the primed quantities by double-primed ones.) It is to be noted that $G^{\prime}$ is polyhedral $\left(\beta_{3}\right)$ and lies interior to $\partial Q^{\prime}$ apart from its boundary.

Thus $\partial Q^{\prime}$ contains two curves $r_{2}^{a}$ and $w^{\prime}$, each bounding a polyhedral disk having only its boundary on the torus. Clearly one disk is in the bounded complementary domain, the other in the unbounded.

7.3. Alexander has proved that a polyhedral torus in a compactified 3space always bounds at least one "solid" torus [1]. By this result and what has just been shown regarding the curves $r_{2}^{a}$ and $w^{\prime}$, it follows that both domains complementary to $\partial Q^{\prime}$ in a compactified 3-space have closures that are solid tori. Hence (Int $\left.Q^{\prime}\right) \backslash G^{\prime}$ is an open 3-cell. In fact, if $G^{\prime}$ is replaced by two disjoint polyhedral disks $G_{1}^{\prime}$ and $G_{2}^{\prime \prime}$ that are close together and both of which lie interior to $Q^{\prime}$ (apart from their boundaries), then (Int $\left.Q^{\prime}\right) \backslash\left(G_{1}^{\prime} \cup G_{2}^{\prime}\right.$ ) is a pair of open 3-cells, $Z_{1}^{\prime}, Z_{2}^{\prime}$, each with a polyhedral 2-sphere as boundary. We may choose $G_{1}^{\prime}, G_{2}^{\prime \prime}$ so that $G^{\prime}$ lies in $Z_{2}^{\prime}$.

Similarly, $\partial Q^{\prime \prime}$ is an "unknotted" torus and hence $Q^{\prime \prime}$ is homeomorphic to a solid polyhedral torus. The definitions of $G^{\prime \prime}, G_{1}^{\prime \prime}, G_{2}^{\prime \prime}, Z_{1}^{\prime \prime}$ and $Z_{2}^{\prime \prime}$ are as above.

7.4. In the following ${ }^{*},{ }^{*}$, and $i$ range over the sets $(a, b),\left({ }^{\prime},{ }^{\prime \prime}\right)$ and $(1,2)$, respectively. The notation was arranged so that $Z_{1}^{*}$ does not meet $G$. Then $U=\left(\right.$ Int $\left.T_{1}\right) \cap\left(\right.$ Ext $\left.T_{2}\right)$ has a closure which is the union of the four 3-cells $\mathrm{Cl} Z_{i}^{*}$. The crucial question is, of course, whether these fit together to form a solid torus.

The boundary of the combinatorial 3-cell $Z_{i}^{*}$ which we denote by $Y_{i}^{*}$ consists of a disk $X_{i}^{*}$ from each of $D_{1}^{a} \backslash D_{2}^{a}, D_{1}^{b} \backslash D_{2}^{b}, C_{1}^{\prime}, C_{2}^{\prime}, G_{1}^{\prime}$, and $G_{2}^{\prime}$. Thus

$$
Y_{i}^{*}=X_{i}^{*}\left(D^{a}\right) \cup X_{i}^{*}\left(D^{b}\right) \cup X_{i}^{*}\left(C_{1}^{*}\right) \cup X_{i}^{*}\left(C_{2}^{*}\right) \cup X_{i}^{*}\left(G_{1}^{*}\right) \cup X_{i}^{*}\left(G_{2}^{*}\right) .
$$

For ease of description we note that the boundary relations of $Z_{i}^{*}$ and the 
disks forming $Y_{i}^{*}$ are those of a cube and its faces. It is also to be noticed that

$$
\begin{array}{lll}
X_{1}^{\prime}\left(D^{b}\right)=\chi_{1}^{\prime \prime}\left(D^{b}\right), & \chi_{1}^{\prime}\left(D^{a}\right)=\chi_{1}^{\prime \prime}\left(D^{a}\right), & \chi_{2}^{\prime}\left(D^{b}\right)=\chi_{2}^{\prime \prime}\left(D^{b}\right), \\
\chi_{2}^{\prime}\left(D^{a}\right)=X_{2}^{\prime \prime}\left(D^{a}\right), & \chi_{1}^{\prime}\left(G_{1}^{\prime}\right)=X_{2}^{\prime}\left(G_{1}^{\prime}\right), & \chi_{1}^{\prime}\left(G_{2}^{\prime}\right)=X_{2}^{\prime}\left(G_{2}^{\prime}\right) .
\end{array}
$$

An examination of the formulas for $Y_{2}^{\prime}, Y_{1}^{\prime}, Y_{1}^{\prime \prime}$, and $Y_{2}^{\prime \prime}$ shows that consecutive pairs of 2-spheres intersect in a pair of disjoint polyhedral disks.

Before discussing the boundaries of the various 2-cells, introduce $m_{i}^{*}(P, Q)$ to mean the polygonal line common to the 2-cells $P$ and $Q$ if $P$ and $Q$ are faces of a cube having an edge in common. Then the boundaries of the above disks (2-cells) are denoted by

$$
\begin{aligned}
& m_{i}^{*}\left(D^{\dagger}, P\right): P=G_{1}{ }^{*}, G_{2}{ }^{*}, C_{1}{ }^{*} \text { or } C_{2}{ }^{*}, \\
& m_{i}^{*}\left(C_{j}{ }^{*}, P\right): P=D^{a}, D^{b}, G_{1}{ }^{*} \text { or } G_{2}{ }^{*}, \\
& m_{i}^{*}\left(G_{j}^{*}, P\right): P=D^{a}, D^{b}, C_{i}^{*} \text { or } C_{2}{ }^{*} .
\end{aligned}
$$

(It is understood if $P=D^{a}$ or $D^{b}$, that we mean the face of $Y_{i}^{*}$ in $D^{a}$ or $D^{b}$ as the case may be.) The 0 -cells may be conveniently denoted by $n_{i}^{*}\left(D^{f}, C_{j}^{*}, G_{j^{\prime}}^{*}\right),{ }^{\dagger}=a, b ; j, j^{\prime}=1,2$. The following set-theoretic relations are easily checked

$$
\begin{gathered}
m_{i}^{*}\left(D^{f}, C_{i}^{*}\right)=X_{i}^{*}\left(D^{f}\right) \cap X_{i}^{*}\left(C_{i}^{*}\right), \\
n_{i}^{*}\left(D^{f}, C_{i}^{*}, G_{i^{\prime}}^{*}\right)=X_{i}^{*}\left(D^{f}\right) \cap X_{i}^{*}\left(C_{i}^{*}\right) \cap X_{i}^{*}\left(G_{i^{\prime}}^{*}\right),
\end{gathered}
$$

with similar formulas holding for $m_{i}^{*}\left(D^{*}, G_{j}^{*}\right), m_{i}^{*}\left(C_{j}^{*}, G_{j^{\prime}}^{*}\right)$, etc.

7.5. The plan to be used in setting up the homeomorphism of $U=\left(\operatorname{Int} T_{1}\right)$ $\cap\left(\right.$ Ext $\left.T_{2}\right)$ and the prototype is as follows: A standard polyhedral plane curve $\Im$ in a Euclidean space $\Re$ is swelled slightly to give a polyhedral unknotted torus. Performing this operation twice we obtain a pair of disjoint unknotted, polyhedral tori $\mathfrak{I}_{1}$ and $\mathfrak{I}_{2}$ with $\mathfrak{I}_{2}$ interior to $\mathfrak{I}_{1}$. The set $\mathfrak{U}=\left(\right.$ Int $\left.\mathfrak{I}_{1}\right) \cap\left(\right.$ Ext $\left.\mathfrak{I}_{2}\right)$ clearly has a closure homeomorphic to $T \times I$ where $T$ is a torus and $I$ a closed number interval. By intersecting this solid with properly chosen planes we obtain a collection of 3-cells whose union will be the range of the homeomorphism. This collection of cells will be isomorphic in a certain sense to the collection composed of the $Z_{i}{ }^{*}$ and their faces. Since the collection of $Z_{i}^{*}$ do not form a complex in the usual sense (let alone a simplicial complex) we use the word isomorphic in a general descriptive sense.

In a Euclidean space $\Re$ let $x, y, z$ denote Cartesian coordinates relative to fixed axes. In the $x-y$ plane let $\Im$ be a closed curve composed of four segments joining the points $(1,0,0),(0,1,0),(-1,0,0)$, and $(0,-1,0)$ cyclicly in order. Let $\mathfrak{T}_{1}$ and $\mathfrak{T}_{2}$ be the polyhedral tori in $\Re$ obtained by swelling (expanding) $\Im$ slightly. We take $\mathfrak{T}_{2}$ interior to and disjoint with $\mathfrak{T}_{1}$. Then the closure of the interior of $\mathfrak{T}_{i}$ meets the $x-z$ plane in a disjoint pair of disks $\mathfrak{D}_{i}^{a}, \mathfrak{D}_{i}^{b}$, where for definiteness we label with superscript $a$ the one lying in 
$x>0$. The circular ring of $\mathfrak{I}_{i}$ contained in $y>0$ is denoted by $\mathfrak{C}_{i}^{\prime} ;$ the one in $y<0$ by $\mathfrak{E}_{i}^{\prime \prime}$. The interior of $\Im$ in the $x-y$ plane is denoted by' $\mathfrak{S}$. The intersection of (S) and $\mathfrak{I}_{i}$ is $\mathbb{Z}_{i}$. Define $\mathfrak{R}_{i}^{\prime}=\mathfrak{S}_{i}^{\prime} \cup \mathfrak{D}_{i}^{a} \cup \mathfrak{D}_{i}^{b}, \mathfrak{R}_{i}^{\prime \prime}=\mathfrak{C}_{i}^{\prime \prime} \cup \mathfrak{D}_{i}^{a} \cup \mathfrak{D}_{i}^{b}$. Thus $\Omega_{i}^{\prime}$ and $\Omega_{i}^{\prime \prime}$ are polyhedral 2-spheres and $\mathfrak{Q}^{\prime}=\mathrm{Cl}\left\{\left(\right.\right.$ Int $\left.\Omega_{1}^{\prime}\right) \cap\left(\right.$ Ext $\left.\left.\Omega_{2}^{\prime}\right)\right\}$ is a polyhedral solid torus ( $\mathfrak{Q}^{\prime \prime}$ is defined similarly).

Then $B \cap\left(\right.$ Int $\left.\mathfrak{I}_{1}\right) \cap\left(\right.$ Ext $\left.\mathfrak{T}_{2}\right)$ is a plane circular ring. Clearly this is divid-

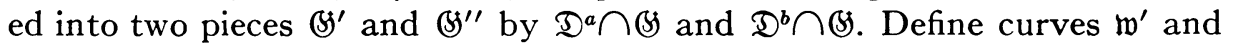
$\mathfrak{w}^{\prime \prime}$ (to correspond ultimately to $w^{\prime}$ and $w^{\prime \prime}$ ) where explicitly

$$
\mathfrak{w}^{\prime}=\left(\mathbb{B}_{2} \cap \Re_{2}^{\prime}\right) \cup\left(\mathbb{B}_{1} \cap \mathfrak{R}_{1}^{\prime}\right) \cup\left\{\left(\mathfrak{D}_{1}^{a} \mid \mathfrak{D}_{2}^{a}\right) \cap \mathbb{S}\right\} \cup\left\{\left(\mathfrak{D}_{1}^{b} \mid \mathfrak{D}_{2}^{b}\right) \cap \mathbb{S}\right\} .
$$

If $(B)$ is moved parallel to itself first above the $x-y$ plane slightly, then below, the positions then occupied by $\mathscr{H}^{\prime}$ will be denoted by $\mathscr{H}_{1}^{\prime}$ and $\mathscr{H}_{2}^{\prime}$ with similar conventions for $\mathscr{S}^{\prime \prime}, \mathfrak{S}_{1}^{\prime \prime}$, and $\mathfrak{B}_{2}^{\prime \prime}$. Then Int $\mathfrak{Q}^{\prime} \backslash\left(\mathbb{S}_{1}^{\prime} \cup \mathfrak{H}_{2}^{\prime}\right)$ is a pair of open 3-cells $8_{1}^{\prime}, Z_{2}^{\prime}$ each with a polyhedral 2 -sphere as boundary. It is arranged that $\mathfrak{B}^{\prime}$ lies in $\mathfrak{B}_{2}^{\prime}$. Corresponding conventions hold for $\mathfrak{S}^{\prime \prime}, \mathfrak{B}_{1}^{\prime \prime}$, $\mathcal{B}_{2}^{\prime \prime}, \mathfrak{B}_{1}^{\prime \prime}$, and $\mathfrak{B}_{2}^{\prime \prime}$. The reader will now verify that if each italic capital in 7.4 representing an $i$-cell, $i=1,2$ or 3 , is replaced by the corresponding German capital, the representation of $i$-cells and the corresponding relations carry over to $\Re$.

One more preliminary is necessary before turning to the proof of Theorem III.

7.6. Lemma. Let $K_{0}$ and $K_{1}$ be disjoint, polyhedral 2-spheres. Let $D_{i}$ and $D_{i}^{\prime}$ be disjoint polyhedral disks on $K_{i}, i=0,1$. Let $x_{i}^{\prime}, \cdots, x_{i}^{n}$ be a set of $n$ polygonal arcs on $K_{i}$ such that $x_{i}^{j} \cap D_{i}=a_{i}^{j}$ and $x_{i}^{j} \cap D_{i}^{\prime}=b_{i}^{j}$ where $a_{i}^{j}$ and $b_{i}^{j}$ are the end points of $x_{i}^{j}$, and $x_{i}^{j} \cap x_{i}^{j^{\prime}}=\square$ if $j \neq j^{\prime}$. If $g$ is a semi-linear homeomorphism of $D_{0} \cup D_{0}^{\prime} \cup \cup_{1}^{n} x_{0}^{j}$ on $D_{1} \cup D_{1}^{\prime} \cup \cup_{1}^{n} x_{1}^{j}$, and $n \geqq 3$, then $g$ has a semi-linear extension $h$ mapping $K_{0}$ homeomorphically on $K_{1}$.

It is clear that if $n \geqq 3$, the homeomorphism $g$ will carry a pair of positively oriented disks on $K_{0}$ onto a pair of like-oriented disks of $K_{1}$ which we might as well take to be positively oriented. A simple application of the Schoenflies theorem to each of the regions on $K_{0}$ determined by $D_{0} \cup D_{0}^{\prime} \cup \cup x_{0}^{j}$ serves to give a homeomorphism of $K_{0}$ on $K_{1}$. Since $g$ is given semi-linear on the boundary of each region, it is known that the extension $h$ can be taken as semi-linear [6].

7.7. REMARKs. The notation above should not be confused with that in the main construction. (The $K_{0}$ and $K_{1}$ above will, in the application to the construction of the main homeomorphism, be contained in the distinct copies of 3-space, $R$ and $\Re$, respectively.)

Although $Z_{i}{ }^{*}$ and $Z_{i}{ }^{*}$ are both combinatorial 3 -cells and hence equivalent under some semi-linear map, we want the additional conditions that $i$-cells of $Z_{i}^{*}$ map onto $i$-cells of $B_{i}{ }^{*}$. 
7.8. Proof of Theorem III. Define $g$ of $n_{2}^{\prime}\left(D^{f}, C_{j}^{\prime}, G_{j^{\prime}}^{\prime}\right)$ to be $n_{2}^{\prime}\left(\mathfrak{D}^{f}, \mathfrak{C}_{j}^{\prime}, \mathfrak{G}_{j^{\prime}}^{\prime}\right)$. Since the symbols $n_{i}^{*}\left(D^{t}, C_{j}^{*}, G_{k}^{*}\right)$ and $n_{i}^{*}\left(D^{* 1}, C_{j_{1}}^{*}, G_{k_{1}}^{*}\right)$ represent the same vertex if and only if the triples $(\#, j, k)$ and $\left(\#_{1}, j_{1}, k_{1}\right)$ are identical, $g$ is 1-1 on the vertices.

Consider the 1-dimensional skeleton of $Z_{2}^{\prime}$ and $3_{2}^{\prime}$. Two distinct vertices of $Z_{2}^{\prime}$ are "related" if and only if their $n_{2}^{\prime}$ representations have precisely two identical arguments. (Thus $n_{2}^{\prime}\left(D^{*}, C_{j}^{\prime}, G_{j}^{\prime}\right)$ and $n_{2}^{\prime}\left(D^{t}, C_{j}^{\prime}, G_{j^{\prime}}^{\prime}\right), j \neq j^{\prime}$, are related and the set-theoretic intersection of the collections of 2-cells determining the 0-cells reduces to a polygonal line $\left.m_{2}^{\prime}\left(D^{t}, C_{j}^{\prime}\right)\right)$. By 7.5 , the corresponding 0 -cells in $\mathcal{B}_{2}^{\prime}$ are related. Thus the function $g$ may be extended to the edges of $Z_{2}^{\prime}$ by requiring the following polygonal lines to be mapped semi-linearly as indicated:

$$
\begin{aligned}
m_{2}^{\prime}\left(D^{A}, C_{j}^{\prime}\right) & \rightarrow m_{2}^{\prime}\left(\mathfrak{D}^{f}, \mathfrak{S}_{j}^{\prime}\right), \\
m_{2}^{\prime}\left(D^{\sharp}, G_{j}^{\prime}\right) & \rightarrow m_{2}^{\prime}\left(\mathfrak{D}^{f}, \mathfrak{S}_{i}^{\prime}\right), \\
m_{2}^{\prime}\left(G_{j}^{\prime}, C_{k}^{\prime}\right) & \rightarrow m_{2}^{\prime}\left(\mathcal{S}_{j}^{\prime}, \mathfrak{\complement}_{k}^{\prime}\right),
\end{aligned}
$$

for $\#=a, b ; j, k=1,2$.

From the boundary relations given in 7.4 and 7.5 it is clear that the boundary of $X_{2}^{\prime}\left(D^{\prime}\right), X_{2}^{\prime}\left(C_{j}^{\prime}\right)$, and $X_{2}^{\prime}\left(G_{j}^{\prime}\right)$ is mapped on the boundary of $X_{2}^{\prime}\left(\mathfrak{D}^{\prime}\right), \mathcal{X}_{2}^{\prime}\left(\mathfrak{S}_{j}^{\prime}\right)$, and $X_{2}^{\prime}\left(\mathbb{S}_{j}^{\prime}\right)$, respectively. Hence $g$ may be extended semilinearly so as to map each of the faces of $Y_{2}^{\prime}$ onto the like-lettered face of $\mathfrak{Y}_{2}^{\prime}$. The extension theorem of Alexander, as extended by Moise and Graeub, gives the desired map of $\mathrm{Cl} Z_{2}^{\prime}$ on $\mathrm{Cl} Z_{2}^{\prime}$.

7.9. Consider $Z_{1}^{\prime}$. Since $\chi_{1}^{\prime}\left(G_{2}^{\prime}\right)=\chi_{2}^{\prime}\left(G_{2}^{\prime}\right)$ and $\chi_{1}^{\prime}\left(G_{1}^{\prime}\right)=\chi_{2}^{\prime}\left(G_{1}^{\prime}\right), g$ is already defined on two faces of $Y_{1}^{\prime}$, which we may refer to as the "top" and "bottom" of $Y_{1}^{\prime}$. There are thus four edges of $Y_{1}^{\prime}$ on which $g$ must be defined to complete the definition of $g$ on the 1-dimensional skeleton of $Y_{1}^{\prime}$. Evidently this extension, as a homeomorphism, can be carried out when and only when the boundaries of $\mathcal{X}_{1}^{\prime}\left(G_{2}^{\prime}\right)$ and $X_{1}^{\prime}\left(G_{1}^{\prime}\right)$ are coherently oriented on $Y_{1}^{\prime}$, then their images on $\mathfrak{V}_{1}^{\prime}$ are coherently oriented. That this is the case is because the images also lie on $\mathfrak{Y}_{2}^{\prime}$ which is a topological image of the orientable surface $Y_{2}^{\prime}$, on which $X_{2}^{\prime}\left(G_{2}^{\prime}\right)$ and $X_{2}^{\prime}\left(G_{1}^{\prime}\right)$ may be coherently oriented. Let the four edges of $Z_{1}^{\prime}$ on which $g$ is not yet defined be given a cyclic order determined by the (unique) end point these edges have in the boundary of $X_{1}^{\prime}\left(G_{2}^{\prime}\right)$. The images under $g$ of these end points gives an order to the vertices of the boundary of $X_{1}^{\prime}\left(\mathcal{S}_{2}^{\prime}\right)$, hence an orientation of the boundary of $\mathcal{X}_{1}^{\prime}\left(\mathbb{S}_{2}^{\prime}\right)$. By the preceding remarks this orientation is coherent relative to that of the boundary of $X_{1}^{\prime}\left(B_{1}^{\prime}\right)$ which is determined by the images of the vertices of the same edges in $X_{1}^{\prime}\left(G_{1}^{\prime}\right)$. Hence the formula

$$
g\left[m_{i}^{\prime}(P, Q)\right]=m_{i}^{\prime}(\mathfrak{B}, \mathfrak{Q})
$$

will be valid if each 1 -cell joining $X_{1}^{\prime}\left(G_{2}^{\prime}\right)$ and $X_{1}^{\prime}\left(G_{1}^{\prime}\right)$ is semi-linearly mapped 
on its unique correspondent in $\mathfrak{D}_{1}^{\prime}$ so as to constitute a (homeomorphic) extension of $g$. Thus $g$ is now defined on the 1 -skeleton of $Z_{1}^{\prime}$ as well as on the "top" and "bottom" of the cube.

The lemma of 7.6 may now be invoked to complete the definition of $g$ on $Y_{1}^{\prime}$. Another application of the semi-linear extension theorem for polyhedra gives a semi-linear homeomorphism of $\mathrm{Cl}\left(Z_{2}^{\prime} \cup Z_{1}^{\prime}\right)$, onto $\mathrm{Cl}\left(\mathfrak{Z}_{2}^{\prime} \cup Z_{1}^{\prime}\right)$.

Consider $Z_{1}^{\prime \prime}$. The boundary $Y_{1}^{\prime \prime}$ meets $Y_{1}^{\prime}$ in a pair of disjoint 2-cells $\chi_{1}^{\prime \prime}\left(D^{a}\right)\left(=\chi_{1}^{\prime}\left(D^{a}\right)\right)$ and $\chi_{1}^{\prime \prime}\left(D^{b}\right)\left(=\chi_{1}^{\prime}\left(D^{b}\right)\right)$ on which $g$ is defined and there are precisely four edges of $Y_{1}^{\prime \prime}$ to which $g$ must first be extended. By the same argument as above (with $Z_{1}^{\prime \prime}$ replacing $Z_{1}^{\prime}$ ), it is shown that an extension to the 1-skeleton of $Z_{1}^{\prime \prime}$ is possible. The lemma of 7.6 is used to complete $g$ on the faces of $Y_{1}^{\prime \prime}$ and finally the extension theorem for polyhedra gives the extension to $Z_{1}^{\prime \prime}$. Thus $g$ maps $\mathrm{Cl}\left(Z_{2}^{\prime} \cup Z_{1}^{\prime} \cup Z_{1}^{\prime \prime}\right)$ semi-linearly onto $\mathrm{Cl}\left(\bigcap_{2}^{\prime} \cup \bigcap_{1}^{\prime} \cup Z_{1}^{\prime \prime}\right)$.

Consider $Z_{2}^{\prime \prime}$. It remains only to define $g$ on two faces of $Y_{2}^{\prime \prime}$ and the interior. (The faces in question are $\mathcal{X}_{2}^{\prime \prime}\left(C_{2}^{\prime \prime}\right)$ and $X_{2}^{\prime \prime}\left(C_{1}^{\prime \prime}\right)$.) Two applications of the extension theorem in the plane and one application of the extension theorem for polyhedra serve to give $g$ as a semi-linear homeomorphism of $\mathrm{Cl}\left(Z_{2}^{\prime} \cup Z_{1}^{\prime} \cup Z_{1}^{\prime \prime} \cup Z_{2}^{\prime \prime}\right)$ onto $\mathrm{Cl}\left(Z_{2}^{\prime} \cup \mathcal{Z}_{1}^{\prime} \cup{Z_{1}^{\prime \prime}}^{\prime \prime}{Z_{2}^{\prime \prime}}^{\prime \prime}\right)$. Thus the required map exists carrying $\mathrm{Cl}(U)$ onto $\mathrm{Cl}(\mathfrak{U})$. This proves Theorem III.

8. Characterization of tame unknotted curves. Let $X$ and $Y$ be topological spaces and $X_{0} \subset X$. If $X_{0}$ has the property that every homeomorphism of $X_{0}$ on itself can be extended to $X$ and if one homeomorphism $h$ of $X_{0}$ onto $Y_{0} \subset Y$ admits an extension $H$ mapping $X$ homeomorphically on $Y$, every homeomorphism of $X_{0}$ on $Y_{0}$ admits such an extension. Combining this observation with the fact that a solid torus is a union of a pair of closed 3-cells, a pair of homeomorphisms of a torus onto the same set both have an extension to the interior of the torus or neither have. If both of the given homeomorphisms are semi-linear and the given extension is semi-linear, the second extension may be taken to be semi-linear.

8.1. Theorem IV. Let $J$ be a simple closed curve in 3-space $R$ having properties $\Phi$ and 2. Then $R \backslash J$ is homeomorphic to the complement of an ordinary circle in 3-space.

Let $\left\{T_{i}\right\}$ be concentric tori in the sense of Theorems II and III so that $\lim T_{i}=J$ and let $\left\{\mathfrak{T}_{i}\right\}$ be concentric standard tori in $\Re$ so that $\lim \mathfrak{T}_{i}=\Im$. Define $U_{0}=\operatorname{Ext} T_{0}, \mathfrak{U}_{0}=\operatorname{Ext} \mathfrak{T}_{0}, U_{i}=\left(\right.$ Int $\left.T_{i}\right) \cap\left(\right.$ Ext $\left.T_{i-1}\right), \mathfrak{U}_{i}=\left(\right.$ Int $\left.\mathfrak{I}_{i}\right)$ $\cap\left(\right.$ Ext $\left.\mathfrak{T}_{i-1}\right), i>0$. Then $\mathrm{Cl} U_{0}$ is a solid, unknotted torus in a compactified 3 -space and by the Alexander theorem may be mapped homeomorphically on to $\mathrm{Cl} \mathfrak{U}_{0}$. By the results of Graeub [6], this homeomorphism may be taken as semi-linear. Let $g$ be such a semi-linear map. Then Theorem III above is applied directly to map $\mathrm{Cl} U_{1}$ onto $\mathrm{Cl} \mathfrak{U}_{1}$ so as to constitute an extension of $g$. Continuing we find 


$$
g\left(\bigcup_{1}^{\infty} U_{i}\right)=\bigcup_{1}^{\infty} \mathfrak{U}_{i}
$$

where $g$ is a homeomorphism of $R \backslash J$ onto $\Re \backslash \Im$.

8.2. The set $\left(a_{1}, a_{2}, \cdots\right)$ is dense on $J$. A dyadic system of notation will be convenient for the proof of Theorem III ${ }_{n}$. Let $a(0)=a_{1}, a(1)=a_{2}$. Let $H$ be one of the arcs of $J$ from $a_{1}$ to $a_{2}$. Let $a_{p_{3}}$ be the first element of $\left(a_{3}, a_{4}, \cdots\right)$ on $H$ and $a_{p_{4}}$ the first on $\backslash H$. Put $a(0,0)=a(0), a(1,0)=a(1), a(0,1)=a_{p_{3}}$, $a(1,1)=a_{p_{4}}$. The set $a_{p_{1}}=a_{1}, a_{p_{2}}=a_{2}, a_{p_{3}}, a_{p_{4}}$ divides $J$ into four sub-arcs. Continuing in a familiar way, at the $n$th stage we have points $a\left(\alpha_{1}, \cdots, \alpha_{n}\right)$, $\alpha_{i}=0,1$, selected from $\left(a_{1}, a_{2}, \cdots\right)$. Every $a_{i}$ first occurs at a unique stage. As before set $a\left(\alpha_{1}, \cdots, \alpha_{n}\right)=a\left(\alpha_{1}, \cdots, \alpha_{n 1} 0\right)$. The disk $D_{n}$ of Theorem II that meets $J$ at $a_{n}$ is denoted by $D\left(\alpha_{1}, \cdots, \alpha_{p}\right)$ if $a_{n}$ is chosen as $a\left(\alpha_{1}, \cdots, \alpha_{p}\right)$.

Let $m_{1}$ be the first integer such that the torus $T_{m_{1}}$ meets both $D(0)$ and $D(1)$. Let $m_{2}$ be the first integer after $m_{1}$ such that $T_{m_{2}}$ meets each $D\left(\alpha_{1}, \alpha_{2}\right)$, $\alpha_{i}=0,1$. In general let $m_{p}$ be the first integer after $m_{p-1}$ such that $T_{m_{p}}$ meets each of $D\left(\alpha_{1}, \cdots, \alpha_{p}\right), \alpha_{i}=0,1$. For our purposes we may replace the sequence $T_{1}, T_{2}, \cdots$ by $T_{m_{1}}, T_{m_{2}}, \cdots$, but retaining the symbols $T_{1}, T_{2}, \cdots$. Then by Theorems II and III, $U_{n}=\left(\right.$ Int $\left.T_{n}\right) \cap\left(\right.$ Ext $\left.T_{n+1}\right)$ has a closure that is a solid torus. The torus $T_{n}$ meets each of $D\left(\alpha_{1}, \cdots, \alpha_{n}\right)$ in a simple closed, polyhedral, curve. The part of $D\left(\alpha_{1}, \cdots, \alpha_{p}\right)$ that meets $\mathrm{Cl}$ Int $T_{m_{p}}\left(\mathrm{Cl}\right.$ Int $T_{p}$ after change of notation) is denoted by $D_{p}\left(\alpha_{1}, \cdots, \alpha_{p}\right)$.

To apply Theorem III, put $Z_{i}^{\prime}=Z_{i}(0), Z_{i}^{\prime \prime}=Z_{i}(1)$. For $n \geqq 2$, $Q\left(\alpha_{1}, \cdots, \alpha_{n}\right)$ is to be that part of $U_{n}$ that is determined by $D\left(\alpha_{1}, \cdots, \alpha_{n}\right)$ and the disk $D\left(\beta_{1}, \cdots, \beta_{n}\right)$, where $\alpha_{1}, \cdots, \alpha_{n}$ and $\beta_{1}, \cdots, \beta_{n}$ are consecutive at the $n$th stage, i.e. order $n$-triples $\left(\alpha_{1}, \cdots, \alpha_{n}\right)$ by $\left(\alpha_{1}, \cdots, \alpha_{n}\right)$ $<\left(\beta_{1}, \cdots, \beta_{n}\right)$ if $\alpha_{j}=\beta_{j}, j<i$, and $\alpha_{i}<\beta_{i}$, in the scale of rational dyadic fractions (modulo 1$)$. Thus $Q\left(\alpha_{1}, \cdots, \alpha_{n}\right)$ meets only the disks $D\left(\alpha_{1}, \cdots, \alpha_{n}\right)$ and $D\left(\beta_{1}, \cdots, \beta_{n}\right)$ at stage $n$.

The pair of 3-cells that $Q\left(\alpha_{1}, \cdots, \alpha_{n}\right)$ is divided into by $G_{1}$ and $G_{2}$ are denoted by $Z_{1}\left(\alpha_{1}, \cdots, \alpha_{n}\right)$ and $Z_{2}\left(\alpha_{1}, \cdots, \alpha_{n}\right)$ where $G^{\prime}$ lies in $Z_{2}\left(\alpha_{1}, \cdots, \alpha_{n}\right)$.

For each $n$ let $P\left(\alpha_{1}, \cdots, \alpha_{n}\right)$ be a family of planes in $\Re$, one for each $\alpha_{1}, \cdots, \alpha_{n}$, through the $z$-axis and dividing the standard torus into congruent parts. The intersection of $P\left(\alpha_{1}, \cdots, \alpha_{n}\right)$ with $\mathrm{Cl}$ Int $\mathfrak{T}_{n}$ is denoted by $\mathfrak{D}_{n}\left(\alpha_{1}, \cdots, \alpha_{n}\right)$. These disks are ordered as are the $D\left(\alpha_{1}, \cdots, \alpha_{n}\right)$. If $\alpha_{1}, \cdots, \alpha_{n}$ and $\beta_{1}, \cdots, \beta_{n}$ are consecutive, the portion of $\mathfrak{u}_{n}$ bounded by $P\left(\alpha_{1}, \cdots, \alpha_{n}\right)$ and $P\left(\beta_{1}, \cdots, \beta_{n}\right)$ that meets no other $P\left(\alpha_{1}, \cdots, \alpha_{n}\right)$ is called a sector of $\mathfrak{u}_{n}$.

8.3. Theorem $\mathrm{III}_{n}$. Let $T_{n}$ and $T_{n+1}$ be polyhedral tori in $R$ subject to the conditions

(a) $T_{n+1}$ lies in the interior of $T_{n}$;

(b) there is a disk $G$ such that $\left(\beta_{1}\right) \partial G=J$ is interior to $T_{n+1}$; 
$\left(\beta_{2}\right) G \cap T_{i}$ is a single curve $s_{i}: i=n, n+1$;

$\left(\beta_{3}\right) G$ is polyhedral modulo $J$;

(c) there is a set of disks $D\left(\alpha_{1}, \cdots, \alpha_{n}\right), \alpha_{i}=0,1$ such that

$\left(\gamma_{1}\right) D\left(\alpha_{1}, \cdots, \alpha_{n}\right) \cap D\left(\alpha_{1}^{\prime}, \cdots, \alpha_{n}^{\prime}\right)=\square$ unless $\alpha_{i}^{\prime}=\alpha_{i}, i=1, \cdots, n$;

$\left(\gamma_{2}\right) D\left(\alpha_{1}, \cdots, \alpha_{n}\right) \cap J=a\left(\alpha_{1}, \cdots, \alpha_{n}\right), a$ point;

$\left(\gamma_{3}\right) D\left(\alpha_{1}, \cdots, \alpha_{n}\right)$ is polyhedral $\bmod J$;

( $\left.\gamma_{4}\right)$ if $D_{n}\left(\alpha_{1}, \cdots, \alpha_{n}\right)=D\left(\alpha_{1}, \cdots, \alpha_{n}\right) \cap\left(\mathrm{Cl} \operatorname{Int} T_{n}\right)$ and $r\left(\alpha_{1}, \cdots\right.$, $\left.\alpha_{n}\right)=\partial D_{n}\left(\alpha_{1}, \cdots, \alpha_{n}\right)$, then $r\left(\alpha_{1}, \cdots, \alpha_{n}\right)$ is a single curve;

$\left(\gamma_{5}\right)$ if $r\left(\alpha_{1}, \cdots, \alpha_{n}\right), s_{i}$ are regarded as 1 -cycles, then $r\left(\alpha_{1}, \cdots, \alpha_{n}\right)$ $\nsim s_{i}, r\left(\alpha_{1}, \cdots, \alpha_{n}\right) \nsim 0, s_{i} \times 0$ on $T_{i}, i=n, n+1$;

(d) $D_{n}\left(\alpha_{1}, \cdots, \alpha_{n}\right) \cap G$ is an arc then $U_{n}=\left(\right.$ Int $\left.T_{n}\right) \cap\left(\right.$ Ext $\left.T_{n+1}\right)$ has a closure homeomorphic to $T \times[0,1]$.

If $n>1, Q\left(\alpha_{1}, \cdots, \alpha_{n}\right)$ is mapped on the sector of $\mathfrak{U}_{n}$ determined by the consecutive $n$-tuples $\alpha_{1}, \cdots, \alpha_{n}$ and $\beta_{1}, \cdots, \beta_{n}$.

The proof of Theorem III $_{n}$ reduces to the proof of Theorem III for $n=1$. For $n \geqq 2$, the region $U_{n}$ is decomposed into $2^{n+1}$ polyhedral 3 -cells $Z_{i}\left(\alpha_{1}, \cdots, \alpha_{n}\right), i=1,2 ; \alpha_{j}=0,1 . \mathrm{Cl} \bigcup_{i} Z_{i}\left(\alpha_{1}, \cdots, \alpha_{n}\right)$ is a solid torus for a fixed $\left(\alpha_{1}, \cdots, \alpha_{n}\right)$. Suppose $g$ is already defined on $Z_{i}\left(\beta_{1}, \cdots, \beta_{n}\right)$ for $\left(\beta_{1}, \cdots, \beta_{n}\right)<\left(\alpha_{1}, \cdots, \alpha_{n}\right)$ so that $Q\left(\beta_{1}, \cdots, \beta_{n}\right)$ maps on the sector of $\mathfrak{U}_{n}$ determined by $\left(\beta_{1}, \cdots, \beta_{n}\right)$. (This condition is omitted if $\left(\alpha_{1}, \cdots, a_{n}\right)$ $=(0, \cdots, 0)$.) Then $D_{n}\left(\alpha_{1}, \cdots, \alpha_{n}\right)$ is mapped on $\mathfrak{D}_{n}\left(\alpha_{1}, \cdots, \alpha_{n}\right)$ semilinearly so that $\mathcal{X}_{i}\left\{D\left(\alpha_{1}, \cdots, \alpha_{n}\right)\right\}$ maps on $\mathcal{X}_{i}\left\{\mathfrak{D}\left(\alpha_{1}, \cdots, \alpha_{n}\right)\right\}$. The edges of $Z_{i}\left(\alpha_{1}, \cdots, \alpha_{n}\right)$ joining the top and bottom of $Y_{i}\left(\alpha_{1}, \cdots, \alpha_{n}\right)$ are then mapped by formulas corresponding to those of 7.8. Lemma 7.6 is used to get the extension to the faces and finally the extension theorem for polyhedrons is used to complete the definition of $g$ on $\mathrm{Cl}\left\{\mathrm{U} Z\left(\alpha_{1}, \cdots, \alpha_{n}\right)\right\}$. If $\left(\alpha_{1}, \cdots, \alpha_{n}\right)=(1, \cdots, 1)$ it is necessary to appeal to the argument of 7.9 to see that the extension to the edges is possible.

8.4. TheOREM V. The homeomorphism $h$ of Theorem IV may be so chosen that for each $n, T_{n}$ maps on $\mathfrak{T}_{n}$ and $D_{n} \backslash a_{n}$ onto $\mathfrak{D}_{n} \backslash a_{n}$.

Proof. By Theorem $\operatorname{III}_{n}, D_{n}\left(\alpha_{1}, \cdots, \alpha_{n}\right)$ is mapped by $g_{n}$ into $D_{n}\left(\alpha_{1}, \cdots, \alpha_{n}\right)$. Since $g_{n+1}$ is an extension of $g_{n}$, it is clear the desired properties for $h$ hold.

Theorem VI. A sufficient condition that an arc or simple closed curve in three space be tame is that it have properties $P$ and 2 . If $J$ is an arc these conditions are also necessary.

Proof. By the remarks of $\S 3$, only the case where $J$ is a simple closed curve need be considered. The necessity of the condition is the content of Theorem I, so let $J$ be a simple closed curve with properties $P$ and 2 . Construction of the sets $\left\{T_{i}\right\},\left\{D_{i}\right\}$, and $G$ is accomplished as in Theorem II, and $h$ is to 
denote the homeomorphism of $R^{3} \backslash J$ onto the complement $\Re^{3} \backslash \Im$ of a standard simple closed curve $\Im$ as defined in Theorem IV.

For each point $p \in J$ and integer $n$ let $\mathscr{D}_{n}(p)$ denote $\bigcup_{1}^{n} D_{i}$ if $p$ lies on none of the disks $D_{1}, \cdots, D_{n}$, while if $p$ lies on $D_{k}$ with $1 \leqq k \leqq n$, let $\mathcal{D}_{n}(p)$ denote $\left[\left(\cup_{1}^{n} D_{i}\right) \backslash D_{k}\right]$. Then let $U_{n}(p)$ be the component of (Int $\left.T_{n}\right) \backslash \mathcal{D}_{n}$ containing $p$, and $\mathfrak{U}_{n}(\mathfrak{p})$ be the correspondingly defined set in $R^{3}$. It is readily seen that $p=\bigcap_{1}^{\infty} U_{n}(p)$, for if $x \in R^{3} \backslash J$ then $x$ lies in no $U_{n}(p)$ with $n>[d(x, J)]^{-1}$, while if $y \in J$ is not $p$ then $y$ and $p$ are separated on $J$ by some pair of disks $D_{s}, D_{t}$ and $y$ lies in no $U_{n}(p)$ with $n>\max (s, t)$. Similarly $\cap_{1}^{\infty} \mathfrak{U}_{n}(\mathfrak{p})=\mathfrak{p}$ for all $p \in \Im$.

Since $h$ carries $T_{n}$ onto $\mathfrak{I}_{n}$ and $D_{n} \backslash a_{n}$ on to $\mathfrak{D}_{n} \backslash \mathfrak{a}_{n}$ by Theorem $\mathrm{V}$ for each $n$, it induces a natural 1-1 set transformation $h_{1}$ of the sets $U_{n}(p)$ onto the sets $\mathfrak{U}_{n}(\mathfrak{p})$. Define $g: J \rightarrow \Im$ by $g(p)=\bigcap_{1}^{\infty} h_{1}\left[U_{n}(p)\right]$, and $f: R^{3} \rightarrow \Re^{3}$ by

$$
f(x)= \begin{cases}g(x), & x \in J, \\ h(x), & x \in R^{3} \backslash J .\end{cases}
$$

Evidently $g$ is a 1-1 transformation of $J$ onto $\Im$ and since $h$ is topological, $f$ is 1-1. To show that $f$ is continuous, it suffices to show that $f$ is continuous at each point of $J$, since $f=h$ elsewhere. As a preliminary step, let $\left\{q_{i}\right\}$ be a sequence of points of $R^{3} \backslash J$ with limit $q \in J$. Then to any neighborhood $\mathfrak{N}$ of $\mathfrak{q}=f(q)$ there corresponds an index $n$ such that $\mathfrak{U}_{n}(\mathfrak{q}) \subset \mathfrak{N}$, since $\bigcap_{1}^{\infty} \mathfrak{H}_{n}(\mathfrak{q})=\mathfrak{q}$. Now $h_{1}^{-1}\left[\mathfrak{u}_{n}(\mathfrak{q})\right]$ is an open set containing $q$ so all but a finite number of the points $\left\{q_{i}\right\}$ lie in this set. Since $h$ is a homeomorphism on $U_{n}(q) \backslash J$ to $h_{1}\left[U_{n}(q)\right] \backslash \Im$, it follows that all but a finite number of the points $\left\{f\left(q_{i}\right)\right\}$ lie in $\mathfrak{U}_{n}(\mathfrak{q})$, so that $\lim _{i \rightarrow \infty} f\left(q_{i}\right)=f(q)$.

Now let $\left\{q_{i}\right\}$ be any sequence of points of $R^{3}$ with limit $q$. Corresponding to each $q_{i}$ there is a sequence $\left\{q_{i j}\right\}_{j=1}^{\infty}$ of points of $R^{3} \backslash J$ with limit $q_{i}$. It has just been shown that for any $i$ the sequence $\left\{f\left(q_{i j}\right)\right\}$ has limit $f\left(q_{i}\right)$, so the sequences $\left\{f\left(q_{i}\right)\right\}$ and $\left\{f\left(q_{i i}\right)\right\}$ have the same limit. But $\left\{q_{i i}\right\}$ must have limit $q$ since $\left\{q_{i}\right\}$ does, so since $\left\{q_{i i}\right\} \subset R^{3} \backslash J,\left\{f\left(q_{i i}\right)\right\}$ has limit $f(q)$.

Thus $f$ is $1-1$ and continuous on $R^{3}$. It must then be a homeomorphism on any compact subset $K$ of $R^{3}$. Taking $K$ to be a solid sphere about the origin containing $J$, this requires that $f$ be a homeomorphism on $R^{3}$ to $\Re^{3}$, and exhibits the tameness of $J$.

Although Theorem VI provides a characterization of tame arcs, a tame simple closed curve obviously need not have property 2 . Since any sub-arc of a tame simple closed curve is tame and hence has property 2, a local form of this property is indicated.

8.5. Definition. An arc or simple closed curve $J$ is said to have property 2 at the point $x$ provided there is a disk $G$ such that

(i) $G$ is locally polyhedral mod $J$,

(ii) $G \cap J$ is an arc, and

(iii) $G \cap J$ is the closure of a neighborhood of $x$ relative to $J$. 
By Theorem I a tame arc has property 2 and hence has property 2 at each point. Since every point of a tame simple closed curve $J$ is an interior point of a tame sub-arc of $J$, it follows that a tame simple closed curve has property 2 at each point. On the other hand, suppose $J$ is an arc or simple closed curve with properties $P$ and 2 at each point, and let $x$ be a point of $J$. Let $G$ be the disk which is locally polyhedral mod $J$ and meets $J$ in an arc $J_{1}$ that is the closure of a neighborhood of $x$ relative to $J$. Since $G$ fulfills the requirements, $J_{1}$ has property 2 , and since property $P$ is hereditary, $J_{1}$ has this property also. Thus, by Theorem VI, $J_{1}$ is tame, so there is a homeomorphism $h$ of $E^{3}$ onto itself such that $h\left(J_{1}\right)$ is a polyhedron. Then if $V$ is a neighborhood of $x$ such that $\mathrm{Cl}(V) \cap J \subset J_{1}$, then $h \mid \mathrm{Cl}(V)$ throws $J \cap \mathrm{Cl}(V)$ onto a polyhedron. This, by definition, means that $J$ is locally tame at $x$, and since $x$ was arbitrary, $J$ is locally tame at each of its points. But, by the result obtained independently by R. H. Bing [4] and E. E. Moise [10], this implies $J$ is tame. The following theorem has been established.

Theorem VII. A necessary and sufficient condition that an arc or simple closed curve be tame is that it have properties $\mathrm{P}$ and 2 at each point.

\section{REFERENCES}

1. J. W. Alexander, On the sub-division of space by a polyhedron, Proc. Nat. Acad. Sci. U.S.A. vol. 10 (1924) pp. 6-8.

2. P. Alexandroff and H. Hopf, Topologie I, Berlin, 1935.

3. L. Antoine, Sur la possibilite d'entendre l'homeomorphisme de deux figures a leur voisinage, C. R. Acad. Sci. Paris vol. 171 (1920) pp. 661-663.

4. R. H. Bing, Locally tame sets are tame, Ann. of Math. vol. 59 (1954) pp. 145-148.

5. R. Fox and E. Artin, Some wild cells and spheres in 3-dimensional space, Ann. of Math. vol. 49 (1948) pp. 979-990.

6. W. Graeub, Die semi-lineare Abbildungen, Sitzungsberichte der Heidelberger Akademie der Wissenschaften (1950) Part N, pp. 205-272.

7. O. G. Harrold and E. E. Moise, Almost locally polyhedral spheres, Ann. of Math. vol. 57 (1953) pp. 575-578.

8. O. G. Harrold, The enclosing of simple arcs and curves by polyhedra in 3-space. Duke Math. J. vol. 21 (1954) pp. 615-622.

9. E. E. Moise, Affine structures in 3-manifolds, V, Ann. of Math. vol. 56 (1952) pp. 96-114.

10. —, Affine structures in 3-manifolds, VIII, Ann. of Math. vol. 59 (1954) pp. 159170.

11. A. Schoenflies, Bemerkungen zu dem vorstehenden Aufsatz des L. E. J. Brouwer, Math. Ann. vol. 68 (1910) pp. 435-444.

The University of Tennessee, KNOXVILle, TenN. 